\title{
A Comprehensive Review on Spectrum Management, Security and Energy-Efficient Cognitive Radio Networks
}

\author{
Shyleshchandra Gudihatti K. N. \\ University Visvesvaraya College of \\ Engineering \\ Bangalore University, Bengaluru, \\ India
}

\author{
S. H. Manjula \\ University Visvesvaraya College of \\ Engineering \\ Bangalore University, Bengaluru, \\ India
}

\author{
Venugopal K. R. \\ Bangalore University \\ Bengaluru, India
}

\begin{abstract}
The explosive growth of mobile devices and the rapid increase in wireless services necessitate an improvement in communication techniques. This technology can boost spectrum utilization as well as meet the massive connectivity requirement to draw attention from academia and industry. Cognitive Radio Network (CRN) is an evident optimal solution in alleviating the challenges of spectrum unused potentially in wireless networks. The Secondary Users are allowed intelligently to access the sections of spectrum unused (spectrum holes) by the authorized Primary Users (PUs). The objective of this paper is to analyze channel throughput, delay, carrier aggregations, energy efficiency, and security challenges and provide research ideas to enhance the overall performance of the CRN. This review paper completely investigated the potential management of spectrum techniques, essentially in spectrum sensing, spectrum allocation, spectrum sharing, and spectrum handoff in CRN. In addition, we study the energy efficient routing techniques like network optimization frameworks in cooperative networks, protocols and different types of attack issues, approaches for handling the secure communication in CRNs. The state of art review, which deeply examines the Cognitive Radio Network providing an idea of important technology and challenges are addressed. The open research challenges related to spectrum management, energy efficient routing and security are discussed.
\end{abstract}

\section{Keywords}

Cognitive Radio Network, Cooperative Network, Energy Efficient Routing, Security, Spectrum Management Techniques.

\section{INTRODUCTION}

These days, wireless communication is steadily growing in automation applications with the increase in wireless equipments like Smartphone's, laptops, tablets and more mobile applications [1][2]. This has led to a tremendous increase in data services leads to exponential increase in data traffic and the ever increasing demand for spectrum resource in wireless networks services. This induces a tremendous attraction of restricted radio resources like bandwidth and energy. In traditional wireless communication systems allocation of spectrum has been implemented for sharing the resources. Even though too many licensed frequency bands, that may result in spectrum under-utilization. According to Federal Communications Commission (FCC) has reported that the spectrum allocated about 70 percent in the United States is not effectively used. As a result, CRNs are envisaged as the key approach to resolve this spectrum crisis to improve spectrum efficiency [3]. The growing data traffic increases the energy consumption in wireless networks. Consider an example, telecommunication data capacity grows by about a factor of 10 every 5 years, which increases the energy consumption by approximately 16 to 20 percent per annum [4].With the ability of CR networks to divert traffic from busy to idle channels, they offer vast applications in the areas of emergency and public safety communications by utilizing the spectrum holes especially in vehicular communications. The emerging spectrum management technologies have enabled CR networks to provide efficient dynamic spectrum access. Application of $\mathrm{CR}$ networks is intelligence assistance, Military and Public Security Applications, Health Care, Home Appliances and Indoor Applications, Bandwidth-Intensive Applications, Real-Time Surveillance Applications, Transportation and Vehicular Networks, and Diverse Purpose Sensing. The Cognitive Network is an advanced Radio Network architecture with promising benefits including cooperative collaboration, dynamic spectrum management and robust cross-layer adaptation capabilities. CRN provides prevalent background knowledge covers wireless communications and cellular networks. A Cognitive Radio is a Radio, changes parameters for transmission purely on interaction with the surroundings it operates [5]. The CR's aim is to seek transmission convenience in the unused (white) spaces and selects the optimal one by means of increasing various utility functions like user's throughput, fairness, etc., without causing zero or meager interference to PUs. Cognitive Radio has two important characteristics:

a) Cognitive capability enables devices to sense their surroundings and choose the optimum transmission technique for the unused spectrum holes.

b) Reconfigurability allows a CR to modify/update its characteristic parameters such as modulation, frequency, etc., and adjust to its surroundings.

In $\mathrm{CR}$, the features are highly important to use the spectrum bands opportunistically which are available, through spectrum hand-off for the PU transmissions are identified. CRs arrangement extends with the Software Defined Radio (SDR) which are devices have Radio capabilities incorporated with programmable features. Presently industries of information and communication technologies worldwide challenges recent development with enhanced Quality of Service (QoS) while parallely reducing its environmental impact. Certainly, there is a requirement of global efficiency both in energy and spectral domains. 


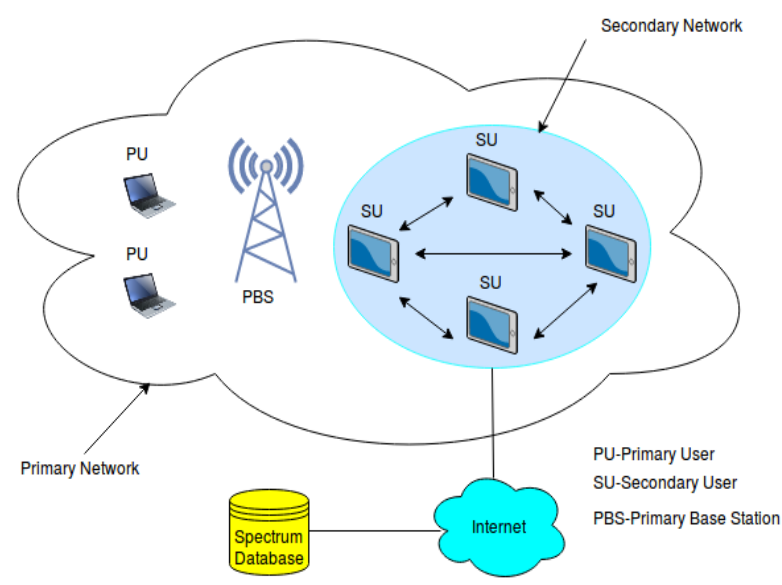

Fig. 1 Cognitive Radio Networks

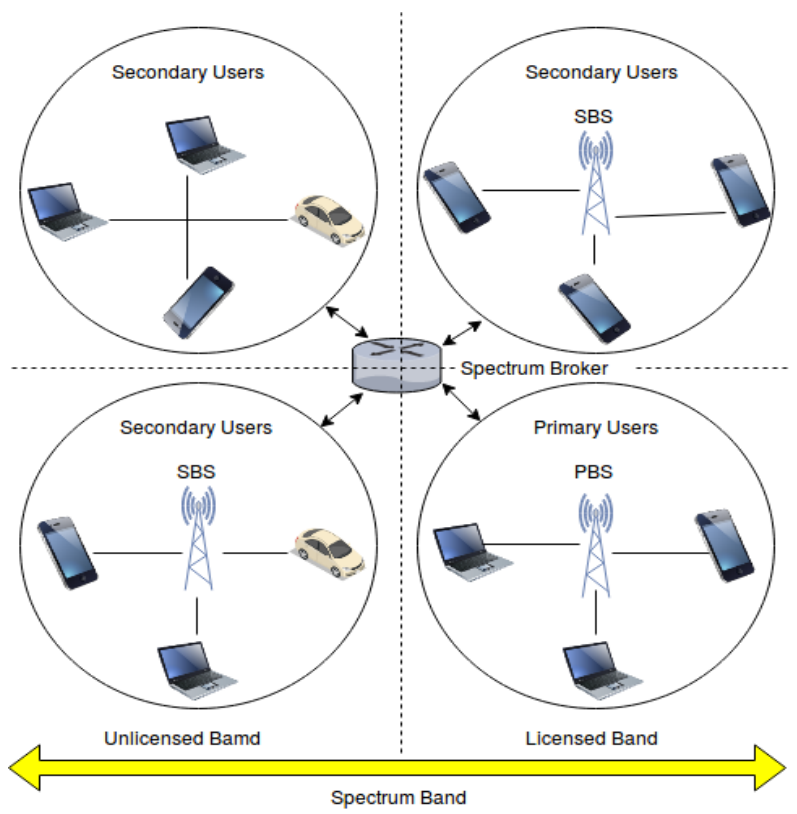

Fig. 2 Cognitive Radio Network Architecture

A typical CRN is depicted in Fig 1. Involves the Primary network consisting of a Primary Base Station (PBS) along with its Primary Users (PUs). The Secondary network with its users (SUs) exists within the Primary network by opportunistically accessing spectrum unused temporarily left by the Primary Users [6]. The spectrum database coexistence with the ubiquitous internet facilitate the activities among networks. The mechanism of sensing based spectrum sharing in (CRN) as shown in Fig 2. The Primary Base Station provides licensed access to the PUs; meanwhile SUs accurately sense the spectrum channel to detect Primary Users idle time for a successful SU transmission. This ensures the spectrum owners (PU) communication is not disrupted. Hence the channels can be classified into licensed and non-licensed bands and a spectrum broker in CRN manages the division and allocation of spectrum between the SUs and PUs. For an undisturbed communication and a comprehensive use of the under-utilized channels, the SUs employ a wide range of sensing techniques and handoff schemes, while consistently trying to manage the desired Quality of Service (QoS) and also preserve privacy.
The Primary functions of CRN are discussed as follows:

- Power Control: Spectrum sharing CR to maximize SUs capacity with power constraints to guard the PUs.

- Spectrum Sensing: It is a technique followed by the Secondary Users to access the channel without interrupting the Primary User transmissions. The SUs detect the spectrum holes by sensing the channel for PU activity and allow transmission upon encountering an idle channel. These sensing techniques may vary from cooperative and noncooperative sensing relies upon whether multiple SUs are involved. It exchanges channel occupancy information or interference based on narrow band and wide band sensing depends on the size of the channel band to be sensed or monitored. Thereby, there are 2 variations of sensing time scales, fast sensing and fine sensing depending upon detailed mechanism of sensing carried out. However, the major problem arises in designing effective sensing devices and formulating efficient algorithms for exchanging spectrum sensing information among nodes/devices.

- Spectrum Sharing: It necessarily deals with the allocation of the available spectrum among various nodes thereby enables a complete utilization.

- Spectrum Analysis: Based on feedback from spectrum sensing is carried out to analyze network characteristics for spectrum vacancies. Its outputs are used for making spectrum decisions.

- Spectrum Decision: This method selects the suitable target channel for an effective transmission without causing interference to other nodes.

- Spectrum Management: Techniques are used to analyze and capture the best available spectrum, which satisfies customer requirements in maintaining the desired QoS without disrupting other users. It also addresses the issues raised during spectrum decision, spectrum sensing and spectrum sharing.

Energy consumption is a major issue, limited processing power, very dynamic topologies, node failures, node mobility, data processing generally required at the node itself, now and again deployed in harsh environments, potentially deployed at very large scale, need to be self-managed. Owing to its exceptional flexibility, Cognitive Radio Networks spans multidisciplinary areas attracting several research works. The challenges however remain numerous; namely, delay/routing overhead, cross-layer architectures, security issues, spectrum management algorithms, and malleable hardware designs. Due to a large collection of published works and multi-faceted concepts, it is an arduous task to provide a comprehensive analysis of all the research tasks. Therefore the objective is to describe the wide research challenges of CRNs. The behavior of a CR can be analyzed by making use of network simulators like OPNET, OMNET, NS2, NS3 and Mat lab etc. These simulators aid in research areas including spectrum sensing, allocation and efficient spectrum utilization. A number of key research motivations are summarized as follows: 
i. Spectrum Policy Alternatives and System Models: The basic underlying idea is to effectively utilize the resource \# management employed in the system.

ii. Spectrum Sensing Algorithms: It is essential for CRN to identify the underutilized spectrum and shares it without any harmful interference to other users. It enables each CRN node to select its operation band without coordination and exchange of control messages with neighbors.

iii. Cooperative Wireless Communications: Its strategies are studied in the presence of channel uncertainty and \# assuming security in physicallayer.

iv. Protocol Architectures for CRNs: CRN specifically focuses from the perspectives of applications, network architectures and protocol design issues.

v. Cognitive Algorithms for Adaptation and Resource Management: It allows spectrum management and sharing in cognitive radio networks (CRN) which could make the spectrum resource management more reasonable.

vi. Network Security for CRNs: CRN covers the recent network security including appliances, threats and policies.

vii. Cognitive Networks and the Internet: Provides next generation of Software Defined Capacity (SDC) for cloud scalable networks and a requirement for cognitive networking.

The contributions of this paper focused on Spectrum Management Techniques, Energy efficient routing methods and Security Approaches. The remainder of this paper is organized as follows. In section 2, an insight into spectrum management techniques namely spectrum sensing, spectrum sharing, cooperative sensing, spectrum allocation and handoff schemes are presented. In section 3, energy efficient routing methods in CRN are focused on energy optimization, resource allocation, power allocation, energy efficient multipath, energy efficiency with harvesting, cooperative and multi-hop networks presented schemes. In section 4, security and privacy in Cognitive networks are explored and conclusions are made in section 5 .

\section{SPECTRUM MANAGEMENT TECHNIQUES}

Today's cellular networks are distinguished by a constant spectrum allocation policy. In addition, large portion of allocated spectrum used infrequently and in a geographical variations. Spectrum usage varies from $15 \%$ to $85 \%$ huge variation with respect to time. Due to the shortage of spectrum vacant and inefficiency of usage requires a new communication model to effectively use the available spectrum opportunistically. Based on the availability of spectrum, Cognitive Radio users communicate with vacant channels without disturbing spectrum owners. We here summarize the reviews in details of different kinds of spectrum management techniques with respect to cooperative sensing, spectrum sensing, spectrum management, spectrum allocation, spectrum sharing, and handoff. Besides we reviewed the latest development took place on spectrum management with its advantages and disadvantages.

\subsection{Cooperative Spectrum Sensing}

This Cooperative Spectrum Sensing (CSS) function is a key idea in CRN. It enables unused spectrum access dynamically and Primary network interferences impact is to be mitigated. However, this approach incurs cooperation overhead, consumption of energy, sensing time and delay increases in heterogeneous networks. These issues are addressed with the latest advances of harvesting energy from the Radio Frequency (RF) signal emitted by Primary Transmitters (PTs). Accordingly, the overall system efficiency is remarkably boosted. The Fig. 3 presents an overview of the cooperative and non-Cooperative Spectrum Sensing methods. In a nonCSS strategy, nodes within a network individually sense the spectrum to identify channel status (idle/busy) and take decisions on its own as per its detection. However, the most simplest and cost effective methods usually used is the energy detection. In a cooperative network, multiple nodes cooperate with each other and exchange information to decide upon the state of the channel. The schemes used are centralized sensing where Fusion Centre is involved as the central agent who decides the channel state. A decentralized scheme where the decision is taken by communicating channel information among the nodes and a hybrid scheme which combines both the schemes.

\subsubsection{Cooperative Sensing:}

To progress the investigation of cooperative sensing, it is to be classified into 3 types based on sharing the sensing data by the cooperating $\mathrm{CR}$ users in the network. They are named as centralized, distributed and relay-assisted are illustrated in Fig. 4. In cooperative spectrum sensing, centralized cooperative sensing Fusion Centre (FC) is the central identity, it controls three step actions cooperative sensing. Firstly Fusion Control chooses a channel/frequency band for sensing and communicates every $\mathrm{CR}$ users to accomplish local sensing individually. All the CR users report the obtained results through control channel, and then FC joins local received sensing data, decides the presence of PUs and radiates the decision reached back to $\mathrm{CR}$ users [7]. As shown in fig 4(a). CR0 is FC and CR1, CR2 and CR3 are cooperating users, which carries out local sensing and reports data back to CR0. In centralized CR network FC is naturally a Base Station (BS), any CR user can work like FC to achieve cooperative sensing and combines with cooperating neighbors. It still faces issues like multipath fading or shadowing and performance analysis is one of the criteria for Realistic Channel Conditions.

Distributed cooperative sensing approach does not depend on FC for the cooperative decision. CR users coordinate themselves to converge collective decision for the presence/absence of PUs by repetition. Thus fig. 4(b) demonstrates the cooperation in a distributed way. The third Approach employs relay assisted cooperative sensing it includes sensing and report channel information. There may be strong sensing channel and weak report channel or viceversa each other to achieve the improvement in cooperative sensing. In fig 4(c) illustrates relay assisted sensing, which 


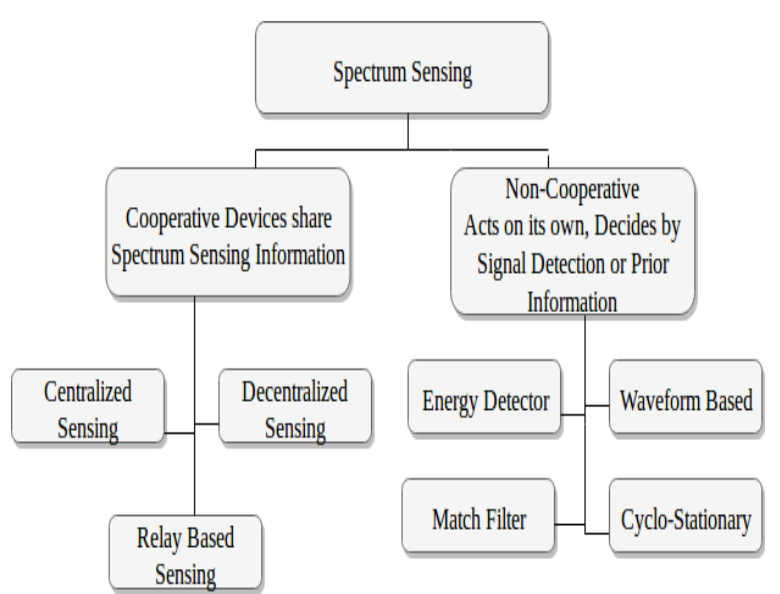

Fig. 3. CR Cooperative and Non-cooperative Sensing Techniques

uses relays to assist in expediting the sensing data. It can also exist in distributed scheme, especially sensing data wants to be forwarded too many hops for arriving the specified receiver node, and each intermediate hops use relays. Thus centralized and decentralized uses only one-hop cooperative sensing but relay-assisted uses multi-hop sensing. In cooperative sensing relays serve for different intention, where CR relays serves for sending the PU traffic.

Components of Cooperative Spectrum Sensing: Cooperative sensing approach commonly treated as a three step process local sensing, reporting, and data fusion. Adding to this, other main elements that are important in cooperative sensing. Here, we identify and summarize the methods of cooperative sensing briefly as follows:

i. Cooperation Models: Cooperative Spectrum Sensing models the CR user how to cooperate and achieve sensing such as parallel fusion network and latest developed game theoretical models.

ii. Sensing Techniques: $\mathrm{RF}$ environment allows to sense, collect samples observed and process the signals for identifying PU signal or spectrum availability.

iii. Hypothesis Testing: Kind of statistical test decides the presence/ absence of a PU. It is performed separately for local decisions/fusion center by each cooperating user.

iv. Control Channel and Reporting: The sensing data of CR users acquired is efficient and reliable to fusion center or mutually shared by CR users via restricted bandwidth and fading-exposed control channel.

v. Data Fusion: It is a method of grouping the shared/reported data for getting cooperative decision. Their data type based on sensing results mixed by decision fusion rule/signal combining approach.

vi. User Selection: CR user deals with how optimally select/decide the correct cooperation footprint/range to increase cooperative gain and reduce cooperation overhead.

Knowledge Base: The information is stored and assists the progress of cooperative sensing method to maximize performance detection. In this case knowledge is either a priori or accumulated through the past experience. The knowledge may involve PU and CR user place of activity, PU activity models, and Received Signal Strength (RSS)
description.Here we discuss some of the open issues in cooperatingModels as follows:

i. Modeling of Cooperation Overhead: The currently available model for cooperative sensing is focused at the detection level efficiency like cooperative gain. A few cooperation overhead problems presented, considers only utility functions for cooperating number of $\mathrm{CR}$ users and throughput of sensing time. Accordingly the cooperation overhead model is still an open challenge.

ii. Modeling of Primary User Cooperation: Existing approaches for cooperative sensing concentrates o the large scale detection of PU like TV base station and predicted that $\mathrm{CR}$ users does not coordinate with PUs. Similarly particular applications areas like military CR network assumptions may not be correct even though PUs may be motivated to communicate with $\mathrm{CR}$ users and connected in adhoc fashion. New model coordinates between PUs and CR users for cooperative sensing and communications. Compressed sensing allows a wideband sensing approach in cooperative sensing. However, still it has lot of open research challenges.

iii. Near Far Problem: A weak signal of PU with nearby strong signal may not be correctly reproduced. It identifies the wideband spectrum owing to sub-Nyquist-rate sampling and shortage of samples. Hence it needs compressed sensing for detection probability.

iv. Implementation Issues: The issues concerned to design achieved by random sampling with the help of compressed sensing. In order to realize these new Analog-to-Digital Converter (ADC) architecture with non-uniform timing and pseudo- random clock generator used. Hence implementations issues for compressed sensing effects need further investigation.

\subsubsection{Spectrum Sensing Efficiency:}

The spectrum sensing proves how frequently allows scheduling to sense cooperatively for suitable number of bands/channels within the specified time. In each round of sensing, it limits on how fast decision reaches. The centralized cooperative sensing refers scheduling performed at the FC and later convergence at distributed cooperative sensing method. Therefore sensing efficiency problems have impact on gain and cooperative sensing overhead.

The purpose of the cooperative sensing is to increase the performance of sensing, which exploits spatially located CR users. This allows more shared sensing information accurately compared to individual decisions leads to cooperative gain. The cooperative gain can be verified from the sensing hardware. Due to shadowing and multipath fading, the SNR of received signal becomes small and difficult to detect the signal. The requirement of sensitivity and limitation of hardware problems can be relieved with the help of cooperative sensing. 


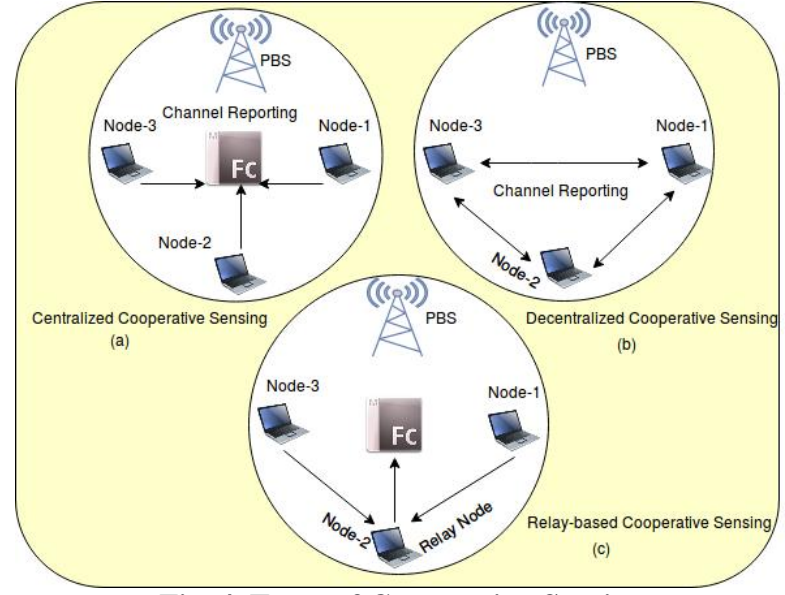

Fig. 4. Types of Cooperation Sensing

The Fig. 5 depicts the cooperation sensing, which presents degrading performance owing to multipath fading and shadowing effect with and without cooperation. In this case consider a Cognitive Radio Network with all the parameters used sensing for the detection of Primary Users with transmit power, path loss exponent, shadowing and multipath effect without cooperation. Similarly same approach applies with cooperation by stating with threshold and without threshold.

The spectrum sensing efficiency problems needs to be Addressed in the future for cooperative sensing:

i. Sensing Scheduling: The maximization of sensing and channel use of one CR user MAC sensing. This scheme envisages how efficiently sense multiple channels to cooperate in CR users.

ii. Analysis of Convergence Rate: The distributed cooperative sensing methods needs to converge for cooperative decision.



Fig. 5. Cooperation Sensing Effect

\subsection{Spectrum Management}

Usually spectrum management deals with four functionalities as shown in Fig. 6 namely, sensing, division or allocation, analysis and mobility. The sensing is carried out in order to accurately identify spectrum vacancies for SU transmissions thereby avoiding interferences with PU. The allocation of spectrum is done to achieve a clear distinction between various users of the spectrum. The analysis of the spectrum is made to ascertain the utilization of the spectrum and to identify spectrum congestion or bottlenecks. The obtained results can then be used for improving spectrum sensing activities as indicated by an upward arrow towards sensing. Spectrum mobility is managed to avail the spectrum access to maximum number of allowed users.

Energy Detection: This is a non-coherent detection, detects the Primary signal purely on the energy sensed. For its uniformity, not necessarily to know PU signal priori information and most reputed sensing approach in cooperative sensing. This technique is often associated with number of disadvantages.

- To achieve probability of energy detection, the time taken for sensing may be high.

- Performance of detection limited to noise power uncertainty.

- Energy detection cannot be employed to separate Primary signals from signals of CR user. Its results to be tightly synchronized and kept away by transmissions during an interval.

- To identify signals of spread spectrum this energy detection never used. Instead of these issues, it remains common detection method in cooperative sensing. Some of the issues related to degradation of performance owing to noise uncertainty can be reduced by diversity gain from cooperation.

a) Primary Signal Detection: The general approach of energy detector measures part of energy in association with received signal on particular time period and bandwidth. It is totally compared with selected threshold value to determine the absence or presence of primary signal [8]. The process of cooperative sensing begins with spectrum sensing performed individually at each CR user named as local sensing. Generally, local sensing for primary signal identification can be formulated as a binary hypothesis problem given as follows:

$$
S(t)= \begin{cases}a(t) & H 0 \\ h(t) \cdot s(t)+a(t), & H 1\end{cases}
$$

where $S(t)$ represents the received signal at the CR user, $s(t)$ represents the transmitted PU signal, $h(t)$ depicts the channel gain of the sensing channel, $a(t)$ represents the zero-mean Additive White Gaussian Noise (AWGN), H0 and H1 represents the hypothesis of the absence and presence of primary user signal in the interest of the PU signal in the frequency band. For the purpose of evaluation of detection performance, the probabilities of detection $\mathrm{P}_{\text {det }}$ and false alarm $\mathrm{P}_{\mathrm{fal}}$ are represented as

$$
\left.\begin{array}{l}
P_{\text {det }}=P\{\text { decision }=H 1 \mid H 1\}=P\{\mathcal{Y}>\lambda \mid H 1 \\
P_{\text {fal }}=P\{\text { decision }=H 1 \mid H 0\}=P\{\mathcal{Y}>\lambda \mid H 0\}
\end{array}\right\}
$$

Where $\mathrm{Y}$ denotes decision statistic and $\lambda$ represents decision threshold. The value of $\lambda$ is adjusted depending on the needs of detection performance. With these definitions, the probability of a miss or miss detection is defined as follows:

$$
P_{\text {mis }}=1-P_{\text {det }}=\mathrm{P}\{\text { decision }=\mathrm{H} 0 \mid \mathrm{H} 1\} .
$$

In cooperative sensing, for evaluating performance of detection probabilities and false alarms of cooperative decisions are represented by $\mathrm{Q}_{\text {det }}$ and $\mathrm{Q}_{\text {fal }}$ respectively.

b) Hidden Primary User Problem: In wireless networking, the hidden node problem or hidden terminal problem occurs when a node is visible to a wireless Access Point (AP), but not to other nodes communicating with 
thatAP. This leads to difficulties in media access control sub layer.

The hidden node problem is very close to Primary User in Carrier Sense Multiple Accessing (CSMA). This causes many ways like multipath fading focused by Secondary Users during Primary Users scanning for transmission. Fig. 6 depicts the idea of hidden node I ssue, the dashed circles represents the operating frequencies of Primary User and CR device. Here, CR devices create interference to receivers as the primary transmitters signal could not be detected due to position of devices [9]. Hence, in literature cooperating sensing is presented to solve the hidden problem of Primary User.

The following section briefly study the techniques used for Spectrum sensing, spectrum sharing, spectrum allocation, andHandoff.

\subsubsection{Spectrum Sensing:}

Cognitive Radio is the solution, which reduces more demand of Radio spectrum. Every node configured with CRs governed by Secondary Users, also aware of the process of Primary Users. Hence SUs dynamically employ the spectrum without disrupting all harmful interference. Conversely to address the more demand of spectrum capability and allowing increased Internet-of-Things applications. Cognitive Radio Networks (CRNs) is envisaged as a key solution to achieve the efficiency of spectrum use. Here we discuss the advantages and disadvantages of the mentioned approaches in details.

Mina et al., [10] introduced a spectrum management distributed learning automata for CR networks, where Secondary Users (SUs) as intelligent agents communicate with Radio Frequency (RF) environment and made appropriate spectrum selection by different feedbacks from environment in a self-organized fashion. This approach ensures less communication delay, interference with Primary Users, and new Secondary Users. The distributed method fails to study admission control problem in CR networks and SUs arrival rates may cross channels service rates. Abdelmohsen et al., [11] modeled a Cognitive Radio framework of spectrum sensing, which presents conventional narrowband and wideband approaches. It guarantees implementation side like complexity, power consumption, throughput, and performance. The next generation CRN like heterogeneous model and cooperation with IoT is not discussed. Hector et al., [12] proposed a spectrum sensing based approach on autocorrelation of samples received, analyzed practically for detection and false alarm probabilities at various Signal-toNoise Ratios (SNRs). It uses Universal Software Radio PeripheralTM (USRPTM) equipments as Radio Frequency. The Euclidean distance approach is more efficient than energy detection with respect to false alarm probability. This work does not focus on kind of noise introduced by USRP equipment, speed measurement and channel scanning to estimate level of utilization.

Jin et al., [13] proposed a Markov chain based greedy channel assignment method (MCGA), where in CRN spectrum sensing and access protocol allows SUs to serially sense the available two channels in a single slot time and provides spectrum opportunities. This approach ensures improvements over the existing methods. However, it fails to focus on sensing multiple channel and non-Bayesian transition probabilities of the channel. Singh et al., [14] studied multihop Cognitive Radio system for performance of spectrum sensing, it uses energy detector and multiple antennas. This approach saves resources of CR, transmission path length by minimizing error rates. The multi-hop approach improves spectrum sensing energy detector to keep the desired Quality of Service (QoS). However, this technique utilizes a large portion of the available bandwidth.



Spectrum Management Functionality

Fig. 7. Spectrum Management

Xiong et al., [15] presented an Adaptive Spectrum Sensing Strategy (ASSS), which determines whether to use Random Spectrum Sensing Strategy (RSSS) or persistent spectrum sensing strategy (PSSS) for spectrum sensing depending upon the estimated PU traffic parameters at a given time period. This strategy allowed using the benefits of both RSSS and PSSS for the different PU traffic patterns and offers more available sub channels for SU transmission. However, ASSS performance depends upon the accuracy of PU traffic parameters estimation to correctly select a spectrum strategy. Wang et al., [16] proposed a single HighOrder Cumulants (HOC)-based spectrum sensing and power recognition scheme, this allows less computation complexity to achieve sensing, power and eliminate uncertainty of noise power. The HOC scheme guarantees multiple orders, develops time lags and increases the performance detection. Nevertheless, spatial diversity of multiple Cognitive Radio sensors is yet to be considered.

Zhao et al., [17] proposed an integrated sequential framework for scheduling wideband sensing, that simultaneously increases sequential detection and compressed

Sensing (CS) mechanism to deliver correct and low cost spectrum sensing. This framework outperforms contemporary methods with respect to sensing delay, overhead and accuracy. Moreover, the accuracy of detecting anomalies achieved by wide band sensing is observed to degrade faster when the presence of anomalies in the received data is low. Cheng et al., [18] proposed a wireless Full-Duplex Spectrum Sensing (FD-SS) and FD Cognitive MAC (FDC-MAC) protocols. The Secondary Users in a multichannel uses non time slotted CRNs to maximize transmission of Primary Users and channel utility of SU without any synchronization between the PU and SU over the multichannel. However, with the FDCMAC protocol, SUs can trade off throughput gained in exchange for high throughput provisioning for PUs. Wei et al., [19] modeled and investigated the bounds of three-regions that provide space time spectrum sensing and access such as black region, grey region and white region. The grey region limits the restriction of false alarm and overlooked detection, while the white region is bounded by analyzing the effect of collective interference from SU to PU. Also black region results from dynamic spectrum leasing. However, the 
performance improvement is minimal during the PU transmission power is low due to rare temporal spectrum opportunities.

Woongsoo et al., [20] proposed the directional antennas deployment in CRNs with centralized cooperative directional sensing scheme. This scheme outperforms other nonoptimized schemes. Nevertheless, false alarms from the sides of PU may be recurrent. Patel et al., [21] developed techniques to address the issues of multi-user Cooperative Spectrum Sensing (CSS) occurring in multi transmitter antennas at each SU and multi receptor at Fusion Center (FC). The Multiple Input Multiple Output (MIMO) Cooperative Spectrum Sensing (CSS) benefits over single user non-cooperative and antenna approaches. Which derives low SNR with and without Channel State Information (CSI) uncertainty of linear complexity? The proposed work of local conclusions prior to transmission and consecutive mixing at the Fusion Center is the future work. Xue et al., [22] investigated the power and spectrum allocation optimization techniques in an Orthogonal Frequency-Division Multiplexing (OFDM) based CR network. The coverage area for the SUs is distinguished into a Hybrid region where they may utilize sensing free spectrum or Underlay access method. In Overlay region SUs adopt Overlay spectrum access schemes. A general Risk Assessment (RA) framework achieves power and channel allocation optimization. For sensing free spectrum access, a novel approach has been formulated with an interference violation test to determine the parameters for the framework

\subsubsection{Spectrum Sharing:}

This section focuses the complete Radio spectrum sharing approach with few techniques performance along with its merits and demerits. Yuan et al., [23] investigated a new model called transparent coexistence, this allows spectrum sharing between Primary and Secondary nodes in a multihop network. The Secondary network opportunistically uses spectrum simultaneously with primary network until it is transparent. This transparent coexistence offers improvement in accessing spectrum and throughput compared to interference avoidance method. Although, a large landscape for practical operation problems needs further research activities. Shuo et al., [24] proposed a joint polarization and power allocation method, which enables spectrum sharing in Cognitive heterogeneous cellular network and takes advantage of spectrum opportunities in polarization and power domains. This method gives better performance compare to spectrum sharing methods in separate polarization, power domain and lower complexity computation than exhaustive search. It is extended to study the spectrum sharing problem between multiple Macro-User Equipments (MUEs) and small networks.

Saha et al., [25] investigated an improvement in multi-user spectrum sharing by using Adaptive Hybrid Relay Scheme (AHR), Authors have observed that outage probability improves with increased number of Primary Users keeping relays and antennas fixed. This scheme increases number of Primary Users compensates the negative impact of high channel rate and achieves a desired level of performance. However, it needs to be extended to another trade-off between number of Primary Users and multi-antenna relays. Wu et al., [26] presented a multi-objective genetic optimization approach, it solves spectrum sharing issues in Underlay sharing model. This approach benefits Secondary network and Primary system interference simultaneously. Moreover, multiple links share the common frequency band. However, spectrum efficiency increased becomes slow with growth of Secondary link power. Adisorn et al., [27] proposed Hybrid spectrum sharing strategy, which includes two heuristic algorithms: static spectrum sharing and dynamic spectrum sharing. These sharing approach are selected adaptively basedon the network status. Thus, it improves overall service satisfaction and better performance tradeoff between achieved

rates and reduced number of handoffs. Thus, this scheme needs to be extended to distribute CRNs and a solution to imperfect sensing is the future work

Nguyen et al., [28] modeled a prediction and sensing based spectrum sharing strategy to enhance detection accuracy in CRNs. In this model, every sensor is redesigned to include both prediction and sensing phases. This scheme is independent with problem size. Jarmo et al., [29] studied a distributed iterative time slot allocation algorithms for Spectrum Sensing Information (SSI), which shares a committed common control channel in a Cognitive Radio ad hoc network. It proves collision-free allocation with probability one if already allocation presents. This approach guarantees fast convergence, higher performance, and small reporting overhead. These algorithms never converges fully in

case of collision-free allocation scenario. Xinxin et al., [30] described the cooperative spectrum sharing approach among multiple PUs and SUs. Which converges to an equilibrium state where all PU proposes a combination of relay power and spectrum access time to attract the SUs, during all SU maximizes its utility by choosing suitable PU. It benefits when

number of SUs is far larger compared to the number of PUs, then its utility losses caused by incomplete partial data and incomplete information are negligible. However, due to more competition happens among PUs, which leads them to reduce their utilities further.

\subsubsection{Spectrum Allocation:}

In Cognitive Radio, spectrum allocation does dynamically to determine the availability of spectrum issues in wireless networks. This technique opens up new problems particularly routing, medium access control and physical layer levels. Many promising solutions proposed to handle these problems and to achieve the wireless network performance uses dynamic spectrum allocation. The main objective is to improve throughput and QoS metrics. However, wireless networks concern is important in energy spent by each node.

Shu et al., [31] presented a channel allocation scheme using graph coloring and rate allocation scheme with a low complexity for multi-hop CRNs. This shows performance in different situations of channel availability, channel allocation and rate allocation schemes which influences end-to-end delay in multi-hop CRNs altogether. It guarantees that the end-to- end delay for the proposed channel allocation scheme is lower than that for the random channel allocation scheme where the channel is allocated randomly. Saleem Aslam et al., [32] proposed a PU behavior aware joint channel selection and allocation scheme for a Cognitive Radio (CR) technology, which solves the problems of spectrum scarcity and low spectrum utilization for Primary Users (PU). This scheme outperforms existing schemes in terms of the transmission time and the number of collisions with the PUs. Hawa et al., [33] designed a fully-distributed algorithm, which offers channel assignment in Cognitive Radio Networks. The idea is to repeatedly leave old bands and join new ones maintaining minimal collisions at the same time by resolving contention in a distributed manner. This algorithm achieves resilience to bandwidth hogs and fairness in sharing spectrum availability with lowest settling time. This algorithm does not depend on dedicated central authority or point of failure. Yousefvand et 
al., [34] designed a new interference constraint capacity aware spectrum allocation. This approach maximizes the CR networks capacity and SUs provided with power control capability. It achieves network capacity higher. Moreover by increasing the number of active links between SUs may not be realistic.

\subsection{Handoff}

Cognitive Radio is introduced to address the scarcity of available spectrum. In order to preserve the desired QoS the Primary Users along with mobility requires spectrum mobility in the network for Secondary Users. By virtue of mobility SU changes its place during transmission, it enters new region with the current channels use by PU. In stochastic nature primary data, any part of traffic may be varying in time, resulting obscured modification for the channel opportunities. To limit the interferences of Secondary Users on the primary transmission during accessing the channel leads to preventing service termination of the Secondary User. Hence user has to empty its channel upon recognition of PU and reconnect other transmission link called spectrum handoff. The schemes proposed for the spectrum handoff can be categorized into two main groups:

i. Slot-based Handoff: In spectrum, handoff technique happens in a time driven manner, selection of channel occurs at start of every time slot with the purpose of increasing throughput of Secondary User.

ii. Connection-based Handoff: Its focus on selected channel for transmission of new connection request on multiple slots and handoff is achieved exclusively when PU backs to the channel.

Ability of CR is the basic characteristic for sensing spectrum, which uses spectrum in an opportunistic fashion. This means $\mathrm{CR}$ has to vacate the spectrum band used if an incumbent signal is identified. Specifically CRs carry out spectrum handoff by finding various spectrum holes for transmission. By doing spectrum handoff often results degradation of $\mathrm{CR}$ accomplishment because more time needs for spectrum sensing. These inherent activities of CRs investigated by opponents one who act like incumbent signals. Several performance issues presented on handoff in CRN along with its merits and demerits.

Maheshwari et al., [35] presented a fuzzy logic based spectrum handoff and assignment approach, which effectively utilizes the channel and reduces channel switching frequently. It ensures network throughput by tolerable limitation of PU interference, signal strength, bit error rate and improves the utilization of spectrum. However, channel quality of PU and SU leads to low utilization of spectrum such as false alarm and miss-detect. S H Manjula et al., [36] demonstrates the AODV routing protocol analyzes the performance of random entity mobility model and pursue cluster mobility model. This mobility model allows less time to transmit the data between each mobility models. Cluster mobility model guarantees to achieve more than random based entity mobility models. However alternative mobility patterns are accustomed to explain practical things is the future works. Ahmed F. Tayel et al., [37] introduced an analytical model for the general case of non-identical channels in CR networks. It provides both fixed and probabilistic sequence approaches for appropriate channel selection. This supports a load balancing model to generate the probability distribution for selecting the target channel, which achieves a good delay performance. A fast hybrid selection approach is suggested for minimum extended data delivery time. However, the extended data delivery time increases as the probability of sensing errors increases.

Alhamad et al., [38] designed a channel reporting scheme based on protocols like Slotted-Aloha (S-Aloha) and Reservation-Aloha (R-Aloha) to minimize channel consignment intricacies. This scheme achieves better performance by limiting the number of reporting slots. Although, R-Aloha outperforms S-Aloha only the mean value of SU reserved slot length is greater. Yan et al., [39] investigated the transmission strategy, Optimal Transmission with Proactive Spectrum Handoff (OTPH) algorithm for Cognitive Radios (CR). This minimizes the total cost and increases the efficiency of data transmission, resolves finitehorizon sequential problem with the help of OTPH and dynamic programming. Although, the total cost for energy consumption incurred increases with an increase in size of data. Yeqing et al., [40] proposed a learning-based and Quality-of-Experience (QoE)-driven spectrum handoff method, that maximizes satisfaction of multimedia users by queuing method to manage the spectrum usage characteristics in CRNs. This queuing approach is effective in prioritizing transmission while avoiding delays due to frequent spectrum handoffs and QoE driven handoff approach increases quality of transmission video. However, SU belongs to a lower priority class that influences higher priority traffic relies on priority-based queuing model. Bicen et al., [41] developed an analytical framework that gauges the effect of Common Control Interface (CCI) integration into Cognitive Radio nodes for spectrum handoff. This framework facilitates the analysis of latent benefits and demerits of CCI for various factors of performance. The inclusion of CCI is not helpful in Cognitive Radio Sensor Network (CRSN) as it elevates energy consumption at low PU traffic usually occurs in sensor nodes. Wenjie et al., [42] presented a sequential sensing based spectrum handoff scheme that reduces total number of spectrum handoffs for CRNs with multiple users. This scheme is carried out by selecting the suitable candidate channels for every SU, and then the best target handoff channel is obtained using Dynamic Programming (DP) based sequential sensing. This scheme provides significant enhancements in system performance by minimizing the spectrum handoffs. DP method reduces the computational complexities meanwhile lowers handoff performance. The following table 1 summarizes the Spectrum Management Techniques concepts/algorithm, advantages and disadvantages. 
Table 1

Spectrum management techniques

\begin{tabular}{|c|c|c|c|c|}
\hline Sl.no. & Authors & Concepts/Algorithm & Advantages & Disadvantages \\
\hline 1. & $\begin{array}{l}\text { Maheshwari et al., } \\
\text { (2015) [35] }\end{array}$ & $\begin{array}{l}\text { Presented a fuzzy logic based } \\
\text { spectrum handoff and assignment } \\
\text { approach, which effectively utilizes } \\
\text { the channel and reduces channel } \\
\text { switching frequently. }\end{array}$ & $\begin{array}{l}\text { Ensures network throughput } \\
\text { by tolerable limitation of PU } \\
\text { interference, signal strength, } \\
\text { bit error rate and improves the } \\
\text { utilization of spectrum. }\end{array}$ & $\begin{array}{l}\text { Channel quality of } \mathrm{PU} \text { and } \\
\text { SU leads to low utilization of } \\
\text { spectrum. }\end{array}$ \\
\hline 2. & $\begin{array}{l}\text { Adisorn et al., (2016) } \\
{[27]}\end{array}$ & Hybrid spectrum sharing strategy & $\begin{array}{l}\text { Improves overall } \begin{array}{r}\text { service } \\
\text { satisfaction and better }\end{array} \\
\text { performance tradeoff between } \\
\text { achieved rates and reduced } \\
\text { number of handoffs. }\end{array}$ & $\begin{array}{l}\text { This scheme needs to be } \\
\text { extended to distributed CRNs } \\
\text { and solutions to imperfect } \\
\text { sensing is the future work. }\end{array}$ \\
\hline 3. & Mina et al., (2017) [10] & $\begin{array}{l}\text { Distributed learning automata } \\
\text { for spectrum management in CR } \\
\text { networks }\end{array}$ & $\begin{array}{l}\text { Incur less communication } \\
\text { delay, interference with } \\
\text { Primary Users, and other } \\
\text { Secondary Users }\end{array}$ & $\begin{array}{l}\text { Fails to study the admission } \\
\text { control problem in CR } \\
\text { networks during arrival rates } \\
\text { of SUs may cross channels } \\
\text { service rates }\end{array}$ \\
\hline 4. & Wang et al., (2018) [16] & $\begin{array}{l}\text { Single HighOrder Cumulants } \\
\text { (HOC)-based approach for } \\
\text { spectrum sensing and power } \\
\text { recognition. }\end{array}$ & $\begin{array}{l}\text { HOC scheme guarantees } \\
\text { multiple orders, develop } \\
\text { time lags and increases the } \\
\text { performance detection. }\end{array}$ & $\begin{array}{l}\text { Spatial diversity of multiple } \\
\text { Cognitive Radio sensors is not } \\
\text { considered. }\end{array}$ \\
\hline 5. & Zhao et al., (2018) [17] & $\begin{array}{l}\text { Integrated sequential framework } \\
\text { for wideband sensing scheduling }\end{array}$ & $\begin{array}{l}\text { Outperforms contemporary } \\
\text { methods with respect to } \\
\text { sensing delay, overhead and } \\
\text { accuracy. }\end{array}$ & $\begin{array}{l}\text { Accuracy of detecting } \\
\text { anomalies achieved by } \\
\text { wide band sensing is observed } \\
\text { to degrade faster when the } \\
\text { presence of anomalies in the } \\
\text { received data is low. }\end{array}$ \\
\hline
\end{tabular}

\section{ENERGY EFFICIENT ROUTING IN COGNITIVE RADIO NETWORKS}

The routing protocol improves with capacity-aware method, that provides conservation of energy and effective data flow between communication nodes. This works with heterogeneous availability of spectrum in centralized and distributed CRN. Much energy efficient routing protocol performs improving conservation of energy, establish the possible paths, and delay minimization with effective utilization of signaling mechanism. Routing methods on CR has been investigated largely in the past.

The secondary network tries to use radio resources which are unused by the primary network. The member of wireless network may share the available radio resource and use of cognitive radio approach avoids collisions and contentions. Dynamic Resource Allocation is an important approach to enhance the throughput of secondary network by optimally using transmits strategies for CRs. In CR bit rate, transmit power, antenna beam and bandwidth allocated dynamically depends upon channel state information available. Here, we discuss in detail on energy efficient CR routing on different aspect. Prathap U et al., [43] discussed to know some of WSN applications, routing algorithms and QoS attributes for the application specific. QoS parameters such as end-to-end delay, packet delivery rate, utilization of energy per packet, overhead routing under changing traffic loads and bit error rates in wireless links used in application domain. However, proposed techniques covers research in a) implement the adaptive routing scheme b) mobility of sensor node in the surveillance domain c) study the attributes of proposed method in the WSN deployment. The routing protocols in CRNs chooses path between source and sink nodes depend on the availabilty of spectrum at each intermediate nodes. If multiple paths are available then the protocol for CRNs uses some metrics which are independent of traffic type to select paths. The Quality of a Service requirement differs based on particular class of data may not be the different data class.

We here summarize the reviews in details of different kinds Of energy efficient routing like energy optimization, power allocation, energy efficient multipath, energy efficiency with harvesting, co-operative networks and multi-hop networks

\subsection{Energy Optimization}

Changle et al., [44] designed a novel method that divides spectrum opportunity into time-frequency slots. It caters to requirements of flexible time-frequency, combinatorial auction method to calculate the best solution of social benefit. The combined approximation method performance is better compared to sorting based greedy algorithm. This novel approach extended to offer SUs to handle multiple bids. Zou et al., [45] studied cooperative CR framework, it addresses dynamic spectrum access and power allocation issues. Here Primary Users employ under-utilized channel and propagate power for Secondary Users to cooperatively relay data. These algorithms guarantees increased aggregate rates of all the SUs. However, the proposed algorithm employs the increasing price adjustment rule.

Zhang et al., [46] developed a distributed boundary estimation algorithm, where SU's to assess the boundary of the no-talk region cooperatively by transferring messages between SUs. This algorithm is robust to SU sensing errors and enhances the throughput of overall system at the cost of boundary estimation. However, the precision for boundary estimation errors remains open besides using heuristic assumptions for the derivation of optimal SU density. Tarannum et al., [47] proposed a Sink administered Load balanced Dynamic Hierarchical Protocol (SLDHP), it increases lifetime of WSNs energy-efficient hierarchical routing protocols. This achieves lifespan of the network by $34 \%$ compared to other and energy consumption reduction of $21 \%$ compared to previous algorithm. SLDHP greatly ensures a load balanced network by hierarchical arrangement of nodes. However protocol for 
applying distributed WSN and enhances the network performance for the present scenario is the shortfall. Amini et al., [48] proposed a new metric termed as Energy per Successful Transmission Time (EPST), it is based on two types of interferences owing to sensing error and obtained from Primary User occupancy again. However, this scheme affects probability collision and average packet delay.

Furtado et al., [49] illustrated towards identifying solutions to attenuate the effect of Spatial False Alarm (SFA), which is responsible for the degradation of CRNs performance and reduces SUs probability medium access. Each SU attempt adopts Energy-Based Sensing (EBS) to summarize the interference originated by much PUs that is placed beyond the desired sensing area. This technique provides a bound for the optimal throughput gained with much PUs and a known level of PU's detection within SU's sensing area in CRN. However, a negligible SFA effect is ensured by increased samples number for sensing accuracy thereby trading off throughput gained by SUs. Yousefvand et al., [50] developed a novel energy spectrum trading idea, this improves energy and spectrum efficiency of radio networks. The enhanced auction model includes algorithms namely, Green Energy Aware Bidding (GEAB) for adaptive bidding and Adaptive Bid Selection (ABS) for bid selection. The proposed scheme enhances the green energy utilization of Secondary base stations by nearly $25 \%$ and reduces power consumption in Primary base stations approximately by $40 \%$. Thus, increased chances of entering sleep modes at low load scenarios. Kiran $\mathrm{K}$ et al., [51] studied a mathematical model, it deals with a hybrid network by splitting traffic decisions made, suitable parameters for routing decisions in a network. This splitting traffic improves throughput and end-to-end delay flow reduces. The routing issue in heterogeneous technologies converge to form interoperable networks is not focused.

Usman et al., [52] introduced an energy efficient process Called Updating and Subset Formation (CUSF) for ad-hoc CRNs. In this process, multiple subsets are formed within a cluster for a single subset, which performs sensing upon selected separate algorithm to allow energy conservation for other subsets. Moreover, all the subsets enter sleep mode during PU active state thereby further reduces power consumption. Michael et al., [53] investigated the case where PU and SU coexist leading to the problem of capacity maximization. They developed an approximation algorithm called CMAX which provides a solution consisting of an optimum set of links from PU and a subset of SU. It enables concurrent transmission without interferences that can disturb the communication. Moreover, in order to enhance performance, a heuristic algorithm is designed based on a greedy strategy. Nevertheless, CMAX underperforms when the path loss exponent is small. Constandinos et al., [54] developed a novel routing strategy incorporated with a traffic based scheme called F-BTD. Its objective is improving energyConservation and promote efficient data transfers among SUs.

This scheme preserves energy by using backward difference of data flow in each node and reduction of a node's active durations. The proposed scheme offers a greater mean throughput as well as enhanced energy utilization. The demerit of this approach keeps the delay per request low.

Rathika et al., [55] proposed QoS Improvement Proper Scheduling (QIPS) model for data traffic situations in all FTP, TCP, HTTP, it offers improved quality of service. This model ensures throughput, efficiency of network and computation complexity of Cognitive Radio Networks. However, this approach needs to study mobile nodes encounter handoffs in Cognitive Radio Network is the future work. Madiha et al., [56] designed to offer a solution to CRSN clustering and routing issue, that uses an energy aware Event-driven Routing Protocol (ERP). So ERP is more reliable, stable and provides better performance in the routing path compared to the protocols used under spectrum aware dynamic data transmission. The theoretical performance analysis is desired and adaptations of different PU spectrum occupation methods investigated to get stronger performance is the future work. Rami et al., [57] proposed two solution concepts that allow stable matching and Walrasian equilibrium. This provides coordination and cooperation procedures to reach them in a distributed fashion. The complexity with respect to average number of suggestions to be low for SU-optimal stable matching. These concepts needs to be devised a technique, which includes the cost incrementing parameter intelligently.

Jianqing et al., [58] developed an energy-efficient cooperative strategy by leveraging temporal and spatial diversity of the Primary network to address energy issues. This scheme enhances SU's energy efficiency compared to existing cooperative schemes. Using this scheme, energy efficiency of SUs is improved by growing number of Pus. The performance is degraded when the packet arrival rate increases. Tsubasa et al., [59] proposed a joint frequency and routing approach that uses prediction of interference for $\mathrm{CR}$ and data rate transmission to reduce end-to-end delay of the SU. It ensures the effectiveness in end-to-end delay time. However, use of many hops that utilizes frequencies of the SU leads to bottleneck in links causes increased end to end delay. Lin et al., [60] proposed new methods Cognitive Transmitter (CT) and Maximum Likelihood (ML), which provides estimation of primary channel gain. This guarantees estimation of errors is very small as 0.015 . Hence, it outperforms the accuracy if the $\mathrm{CT}$ is weak for primary signals sensed

Mihir et al., [61] devised hard and soft reports algorithms based on Gaussian mixture model, this differentiate sporadically transmitting Incumbent Users (IU) and identify the CRs receiving signals from every IU. The devised scheme minimizes the computational complexities. Thus, the detection performance is degraded due to channel capture effects. Huijin et al., [62] introduced a Markov Decision Processes (MDP) method to achieve a minimum delay performance based on the examination of queues. In order to minimize the MDP problem complexity, a myopic algorithm is developed which provides an enhanced performance for delay sensitive and best effort services. Still, the scheme cannot support larger number of channels. Haijun et al., [63] discussed the cognitive small cell networks, allows to curb the increased mobile traffic. Which analyze joint sub channel and power allocation issues raised in cognitive networks. A cooperative Nash bargaining game, that guards Primary macrocell, enables reliable transmission through reduced outage probability. Moreover, a low rate demand is needed for intra-small cell fairness capability and achieves better tradeoff between capacity and complexity. However, this system assumes only a multi-sub channel network.

Piyush et al., [64] introduced the constrained resource allocation issues that grow in the circumstance of spectrum sharing in CRNs for effective utilization of multi-dimensional formulation. There are three tasks for SUs are power assignment, frequency and directionality of antenna, which achieves variations of NP-hard optimization. This novel methodology provides multiple directions for future work as practically important and extended to work CRN design, 
implementation and QoS limitations. Zhang et al., [65] introduced a resource allocation technique composed of two algorithms, namely spectrum sensor scheduling and data sensor resource allocation algorithms to achieve endurance in

Spectrum sensors and minimize energy consumption in data sensors. This technique enables an efficient usage of Primary network channels and optimizes scarce resources allocation in battery-powered data sensors.

\subsection{Power Allocation}

The power allocation (PA) problem is investigated in Cognitive Radio Networks (CRNs) that uses Non-Orthogonal Multiple Access (NOMA) methods. In such a framework, Quality of Service (QoS) requirements are met for Primary Users (PUs) and Secondary Users (SUs). This paper focuses on analyzing power allocation between channels in a cognitive wireless network for Primary Users, which uses spectrum underlay method.

Hecke et al., [66] derived a beamforming and optimal power allocation strategy, which reduces the outage probability of a cooperative SU network. Only with the support of multiantenna AF-relay, it preserves the QoS of PU networks. Nan et al., [67] proposed three algorithms namely Power Allocation algorithm which Maximizes Rate of Secondary Users (SUs) (PAMRSU), Maximizing the Energy Efficiency of the Network (PAMEEN) and Maximizing satisfaction of SUs (SSU) (PAMSSU) for Interference Alignment (IA)-based $\mathrm{CR}$ networks. These power allocation methods minimize transmitted power of the PU and improve the performance of Primary User. However, it needs to be carefully allocated to guarantee the QoS of the PU. Hang Hu et al., [68] presented a Joint Spectrum Sensing and Power Allocation (JSS-PA) approach, which improves spectrum efficiency with the aid of information location of Primary Transmitter and CR network. JSS-PA technique outperforms Only Spectrum Sensing (OSS) and Power Allocation (OPA) scheme. The Spectrum Efficiency of Spectrum in Soft Information Fusion (SIF) method is greater compared to Hard Information Fusion (HIF) strategy. However, enhancing accurate estimation of the protected region is the future work.

\subsection{Energy Efficient Multipath}

Kishor et al., [69] introduced an Energy-Efficient and Reliable Multipath Routing protocol ( ERMR ) for CRAHNs. Due to its dynamic nature, unexpected behavior allows energy efficiency and reliability in CRAHNs. This method ensures throughput of network, packet delivery ratio and average energy. It fails to consider the work for mobility of PUs. Zhutian et al., [70] designed a new RPL-based routing protocol (Green-RPL) that proves energy efficiency over virtual distance (EEVD) by considering the important factor of routing. Multiple neighbor nodes are chosen during a single hop to structure a forwarder set and meets QoS requirements of Cognitive Radio Automated Metering Infrastructure (CRAMI) network. This ensures protection of PU's and improves energy efficiency significantly. In this approach, benefits of Green-RPL lowers due to higher node density environment and closes the enhancement of other protocols. Jie et al., [71] proposed a novel anypath routing Spectrum Aware Anypath Routing (SAAR), this routing mechanism considers CRNs spectrum uncertainty and affected transmission behavior of wireless channel. SAAR ensures packet dropping ratio, endto-end delay and throughput. This method fails to study implementation of distributed Cognitive Radio Networks. Weiqiang et al., [72] designed an energy efficient cross layer optimization for multihop Multiple-Input Multiple- Output
(MIMO). CRN design enables wireless communications, which balances network utilization, weighted total power use of SU communication with minimum PU transmission rate and the SU power constraints. The proposed method provides good performance and power-efficient while managing the high network utility. Thus, total traffic rate of all flows moving across a link cannot exceed the capacity. Ghalib A et al., [73] proposed a cluster-based solution that addresses the issues of multimedia scheduling in Cognitive Radio Sensor Network (CRSN). Clustering is explored to maintain dynamic spectrum access and QoS routing in multimedia CRSN. This optimal clustering approach offers desired QoS in energy efficient delivery for multimedia and incurs three times lesser delay with respect to higher deliver ratio. It needs to be focused on convergence of video quality. Prakash et al., [74] proposed Residual Energy Adaptive Re-Routing (REAR) method with a Mixed Integer Linear Programming (MILP) model for increasing the network lifetime. This approach supports multi-commodity flow where multiple sources to sinks network requests involves splitting and re-routing over multiple paths. The energy use greatly reduces under high loads. REAR approach benefits common energy consumption across each nodes in the network therefore network lifetime increases.

\subsection{Energy Efficiency with Harvesting}

Ahmed et al., [75] developed a novel strategy to combine varied traffic design and formulate a transmission of threshold-based SU. Which optimizes its transmission as well as enhances prospects of harvesting energy? The work focused only on a single SU pair and a single PU channel. Tarannum et al., [76] advised an algorithm of Energy Efficient Routing Protocol (EERP) suitable for energy constrained sensor networks. The idea of new proposed protocols that uses lowest energy path outcomes in long-term fitness of sensor network. This shows a noticeable enhancement of $11.4 \%$ network lifetime. Consequently this decreases the energy utilization per node in comparison to Directed Diffusion Protocol (DDP) and consistently achieves energy-based metrics, altered shortcut path method to send data provides a robust mechanism. However, allowing the routing model for extending in dynamic sensor networks is the future work. Zan et al., [77] proposed to improve the spectrum energy efficiency and energy harvesting schemes in CRNs. The frameworks of Markov Decision Process (MDP) decide the balanced spectrum sensing. It ensures transmit energy and spectrum sensing interval to maximize throughput of SU. Thus, this scheme significantly improves the average throughput only by trading off interference caused to PU. 


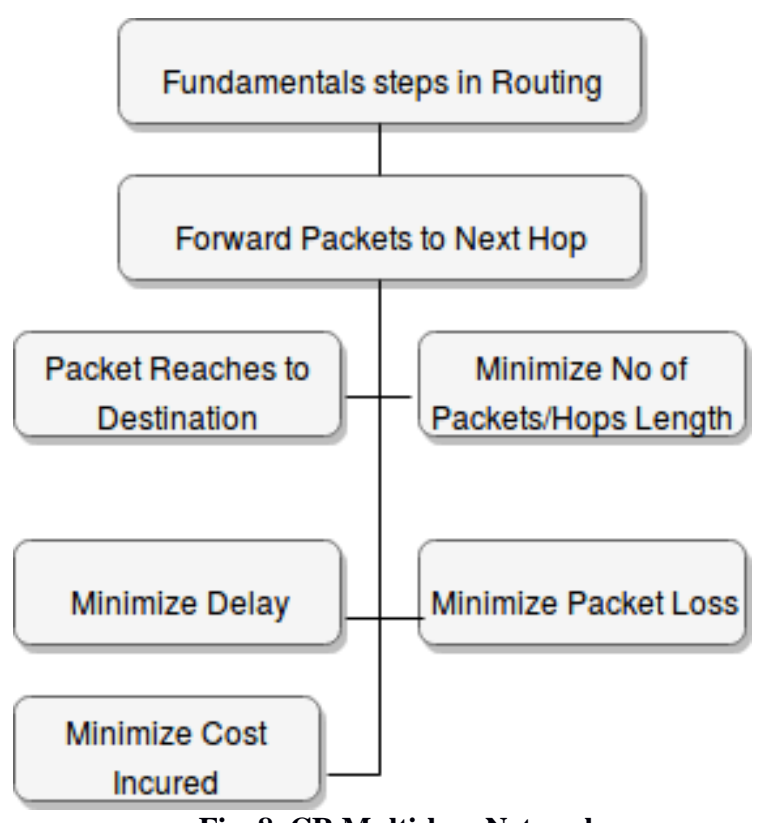

Fig. 8. CR Multi-hop Network

Kanavalli et al., [78] studied a flat routing protocol, which is used in wireless sensor networks. This approach allows route finding process and total messages in a network. Hop count used to determine route from source to sink and energy of neighbor nodes for every node. This protocol performs much better compared to flood routing protocol. It scales well and enhances lifetime of network by controlling the total messages which are forwarded through the network and accomplishes a high level of energy efficiency. Xing et al., [79] studied two techniques for the enhancement of spectrum efficiency and energy harvesting in cooperative CRNs namely, dedicated Energy Beamforming (EB) and Full-Duplex (FD) relaying by multi-antenna energy access points. This technique assumes a two equal slot Decode and Forward (DF) relaying to resolve the problem of low efficiency of wireless power transfer. Thereby, it increases low cooperation efficiency.

\subsection{Co-Operative Networks}

The aim of this section is to gather recent research contributions and advances in Cooperative Cognitive networks (CCNs). In CCNs, there exists collaboration among the Primary and Secondary systems (Transmitter \& Receiver pair) in the sense SUs are offered spectrum access in return for relaying data between Primary Transmitter (PT) and PU by the Secondary Transmitter (ST) subsequently utilizing the spectrum proficiently.

Vahidian et al., [80] studied performance of bidirectional Cognitive cooperative networks, it allows the interference from Secondary transceivers to PU. Due to the interference presence at Secondary nodes becomes error floor phenomenon in the outage and Bit Error Rate (BER) accomplishment. Thus, the Secondary Network (SN) guarantees full diversity order in all cases. However, the number of relays used is only one. Arsany et al., [81] proposed a cross layering routing protocol, that combines physical layer methods in routing layer. This technique effectively utilizes cooperative groups and employs beamforming to send data even though Primary Users exists. It achieves low overhead and marginal end-to- end delay. Thus, it is assumed that PUs is stationary and extended this work for mobile PUs. Raikel et al., [82] formulated the statistical CSI based Power Allocation (PA) (SCPA) scheme as an optimization problem based on the approximate outage probability expression and its convexity. Network-Coded (NC) scheme presents considerable advantage over the DF and Direct Retransmission (DT) schemes in terms of power consumption and outage probability. It is limited completely for non-symmetrical network geometry.

Shuyu et al., [83] proposed a Spectrum Aggregation-based Protocol for Cooperative Routing in CRAHNs. SACRP has two classes of routing approaches, in which class A and class $\mathrm{B}$ offers power minimization or throughput maximization, and reduces end-to-end delay. These classes achieve reduced retransmissions with help of relay nodes and end to end delay. Classes A and B needs to investigate the applicability of network reliability and receiver protection of PU. Yuan et al., [84] proposed a sub-Nyquist wideband spectrum sensing method, which identifies occupied channels without notice. By obtaining signal support depends on sparse nature of multiband signals. This method achieves performance detection, reduces computation and implementation complexity compared to cooperative spectrum sensing approaches. However, it lacks in identifying the active channel without noticing prior knowledge of received spectrum signal either sub-Nyquist sampling or reconstruction

Stage. Jiang et al., [85] proposed a new cooperative algorithm that constructs associative routing in multi-hop cognitive networks with multiple primary and Secondary Users. This method assumes interference among Primary Users and Secondary Users. This algorithm achieves lower energy consumption of a network. However, transmission power increases with increased number of Secondary Users. Yanqing et al., [86] a cooperative game is formulated, which solves spectrum sharing issue for multiple Secondary Users in a MIMO cognitive radio network. An efficient distributed method is developed, that converges rapidly optimal solution with moderate signaling in the network. Multiple Input Multiple Output CRN consideration improves solution is the future work.

\subsection{Multi-Hop Networks}

Multi-hop networks employ relays between nodes for the purpose of communication when the coverage area is greater than the range of single nodes. Since the nodes are power constrained, multiple hops greatly improve the energy efficiency especially for long distance transmissions. The fundamental step of a multi-hop routing scheme is as shown in Fig. 7. Where the major criteria while hopping packets include minimization of incurred expenses, count of intermediate hops taken to arrive the destination, number of packets lost during transit, transmission delay and delivery to the correct destination/intermediary receiver.

Dingde et al., [87] proposed an optimal multicast communication approach, which provides lowest energy consumption for wireless multi-hop networks with restricted energy and directional reception antenna model. It constructs the best multicast paths as per the directional reception of double step reconstruction routing. It significantly improves network lifetime and power transmission. This fails to focus on cross-layer network. Yi et al., [88] introduced a broadcast protocols performance analysis for multi-hop CR ad hoc networks. To address these challenges a new unified analytical

approach is developed and analyzed the broadcast protocols with any topology. This methodology in traditional MANET cannot use CR ad hoc networks and fails to indicate unique characteristics in multi-channel multi-hop ad hoc CR network. Dibakar et al., [89] developed two multi-hop ad-hoc networks, That uses IEEE 802.11 based MAC protocol. It allows variant 
channel priority of access and probabilistic routing protocol considered ideal sensing process. This approach ensures average end-to-end delay and achieves throughput. It needs to be analyzed for multiple channels and non-ideal sensing process.

Avik et al., [90] formulated an optimization problem in multihop CRN, which minimizes end-to-end Secondary outage probability considering the limitations of energy causality and rate of PU cooperation. This guarantees a stable network having less chance of outage during transmission of Secondary data and results minimum value of PS. It needs to be extended for the existence of multiple eavesdroppers. Qingkai et al., [91] investigated the spectrum-mobilityincurred that offers route-switching issue in multihop CRNs. It studies both reassignment of channel and rerouting. This ensures theoretical tests and illustrates tradeoffs between routing and switching costs. It fails to study the channel

Table 2

energy efficient routing protocols techniques

\begin{tabular}{|l|l|l|l|l|}
\hline Sl.no. & Authors & Concepts/Algorithm & Advantages & Disadvantages \\
\hline \hline 1. & Shuyu et al., (2015) [83] & $\begin{array}{l}\text { Spectrum aggregation-based protocol for } \\
\text { cooperative routing in CRAHNs }\end{array}$ & $\begin{array}{l}\text { Class A achieves reduced } \\
\text { retransmissions with help of } \\
\text { relay nodes and end to end } \\
\text { delay }\end{array}$ & $\begin{array}{l}\text { Needs to investigate the } \\
\text { applicability of network } \\
\text { reliability and } \\
\text { receiver } \\
\text { protection of PU. }\end{array}$ \\
\hline 2. & Hang et al., (2016) [68] & $\begin{array}{l}\text { Joint Spectrum Sensing and Power } \\
\text { Allocation (JSS-PA) approach, that } \\
\text { improves spectrum efficiency with the } \\
\text { aid of information location of Primary } \\
\text { Transmitter and CR network. }\end{array}$ & $\begin{array}{l}\text { The Spectrum Efficiency } \\
\text { of Spectrum in Soft } \\
\text { Information Fusion (SIF) } \\
\text { method is higher compared } \\
\text { to the Hard Information } \\
\text { Fusion (HIF) strategy. }\end{array}$ & $\begin{array}{l}\text { However, } \\
\text { accurate estimation of the } \\
\text { protected region is the } \\
\text { future work. }\end{array}$ \\
\hline 3. & Jie et al., (2017) [71] & $\begin{array}{l}\text { Novel anypath routing mechanism } \\
\text { Spectrum Aware Anypath Rouitng } \\
\text { (SAAR) }\end{array}$ & $\begin{array}{l}\text { SAAR ensures packet } \\
\text { dropping ratio, end-to-end } \\
\text { delay, and throughput. }\end{array}$ & $\begin{array}{l}\text { It needs to study } \\
\text { implementation } \\
\text { distributed } \\
\text { Radio Networks. }\end{array}$ \\
\hline 4. & $\begin{array}{l}\text { Kishor et al., (2017) } \\
{[69]}\end{array}$ & $\begin{array}{l}\text { Energy-Efficient and Reliable Multipath } \\
\text { Routing protocol ( ERMR ) for } \\
\text { CRAHNs. }\end{array}$ & $\begin{array}{l}\text { Ensures conventional } \\
\text { protocol such as throughput } \\
\text { of network, packet delivery } \\
\text { ratio and average energy. }\end{array}$ & $\begin{array}{l}\text { Fails to consider the work } \\
\text { for mobility of PUs. }\end{array}$ \\
\hline 5. & $\begin{array}{l}\text { Zhutian et al., (2017) } \\
{[70]}\end{array}$ & $\begin{array}{l}\text { Designed a new RPL-based routing } \\
\text { protocol (Green-RPL) for energy } \\
\text { efficiency over virtual distance (EEVD) }\end{array}$ & $\begin{array}{l}\text { It guarantees protection of } \\
\text { PUs and improves energy } \\
\text { efficiency significantly. }\end{array}$ & $\begin{array}{l}\text { Benefits of Green-RPL is } \\
\text { greatly lowers in higher } \\
\text { node density environment } \\
\text { and closes the enhancement } \\
\text { of all other protocols. }\end{array}$ \\
\hline
\end{tabular}

Switching costs of spatial and frequency domains for CRNs. The following table 2 summarizes the Energy Efficient Routing Protocols concepts/algorithm, advantages and disadvantages.

\section{SECURITY}

Security refers to a measurement of digital information either internal or external malicious users. It ensures only authenticated people to access the available personal information. Security is free from or resilience against harming unknown forces. Beneficiaries like persons, social groups, objects, institutions, ecosystem and other entities which are vulnerable to change by its environment. Here, Cognitive Radio spectrum sensing is a prerequisite to ensure Secondary Users to rescue from interference with Primary Users while accessing the vacant band. However, during this process security issues like eavesdropping means untrusted users (Secondary) may try to decode message by sensing information without authentication. Always security is a burden in wireless network, in which Primary Users requires secured communication in CRN. Traditional approaches use encoding methods in upper layers but it is compromised and becomes a challenging task without infrastructure for a network. Since the cooperative CR users major concern is reliability and security when many $\mathrm{CR}$ users sensing cooperatively. Owing to this, users may report effected or falsified sensing information influences cooperative decision. Sometimes malfunctioning of CR users unknowingly sends unreliable information to the Fusion Center (FC). Moreover, intentionally CR users (malicious) manipulate sensing data and list the falsified information for further use. For example, these malicious users get spectrum access falsely by reporting PUs presence. The cooperative gain caused by malicious CR users by sensing cooperatively. To address issues like reliability and security, new approaches are necessary to detect malicious users, manipulated data and cancel the cooperative sensing. Hence, it encounters cooperative sensing overhead, needs to guarantee secure operations and obtain reliable results in hostile surroundings.

In addition to falsification of data issue, there is variety of security attacks vulnerable during cooperative sensing. Essentially PU emulation attack, jamming attack in control channel and attack node capture. These malicious users send the signals similar to PU, falsely identified as PUs and valid $\mathrm{CR}$ users vacate the frequency band, attacker has wrongful privilege to spectrum access [92]. In order to address the above mentioned issues require verification of transmitter localization to handle the attack. The localization is effectively utilized by grouping the Received Signal Strength (RSS) values for cooperating CR users to calculate location of PUs transmitter. Its identity can be cross checked by comparing the calculated values of known PUs behavior. For jamming attack, a very strong interference signal applies to control channel which disables reception of correct control message of $\mathrm{CR}$ users. The $\mathrm{CR}$ users not in position to mutually change the control message in cooperative sensing and functions of higher level network results in Denial-of- 
Service (DoS). The jamming attacks can be lowered with spread spectrum approaches by allowing pseudo random access to unidentified malicious users. We present a comprehensive list of major known security threats and attacks, vulnerabilities, software defined security in a Cognitive Radio Network (CRN).

\subsection{MAC Layer and IEEE 802.22 Specific Threats}

MAC Layer Threats: It is associated with physical layer strictly along with hardware equipments plays an important role in protecting the interference to PUs in CRNs. In CRs communication interaction between layers does not follow strict separation of layers in basic TCP/IP protocol stack. There are mainly two kinds of protocol present namely IEEE 802.22, application specific protocol. IEEE 802.22 is a MAC layer CRN based infrastructure where application specific protocol is CR ad-hoc networks. CR-MAC protocols deployed in distributed CRNs, which support cognitive capacity without any objects use. Common Control Channel (CCC) plays important characteristics for those protocols. It supports conditional functionality in CRN and also becomes target to cause DoS attacks.

Common Control Channel Threats and Vulnerabilities: CRs exchange control information with the help of CCC. The control messages are transmitted with predetermined channel frequency, belongs to out of band channel, and different from the actual data exchange. It is used for exchanging many type of information such as channel negotiation, collaborative sensing, handoff etc. The protection of CCC is required first otherwise tries to compromise. If they are successful, its performance will be affected in various ways since it belongs to the main process of managing the operations of a network. Here below lists some of the threats faced by CCC:

a. MAC Spoofing: Usually attackers propagate spurious reports, which aim to cancel the activities of CRN. This type of attack is vulnerable to Multiop CRNs, clearly no central control for authentication between nodes and data integrity protection.

b. Congestion Attacks: This type of attackers distributes CCC to affect DoS attack.

c. Jamming Attacks: Normally attackers bring out DoS attacks at physical layer by developing interference.

\subsection{Cross-Layer Attacks}

Already, we studied threats and detection types only on single-layer analysis and methods [93]. Primary User Emulation Attacks (PUEAs) and Spectrum Sensing Data Falsification (SSDF) attacks concentrates on physical layer while MAC threats aims on MAC layer. However, opponents launch attacks aims to multiple layers effects entire cognitive cycle and becomes feasible to all layers. Mainly attacks are classified into two types to identify cross layer attacks such as i) At the physical layer, hypothesis test used to identify SSDF attacks and ii) Identify the adversaries aware of allocating back off window size to detect at MAC layer and compares the expected distribution.

\subsection{Software-Defined Radio security}

CRs provide reconfigurability realization done with the use of SDRs, its protection is most important from malfunctioning by attackers. SDRs protection classified into two types namely software and hardware based protection. Software based approaches contains deployment of tamper-resistance methods to protect against malicious users. These algorithms involves secured download and software distribution into various SDRs. CRNs are flexible, software updation downloaded frequently to defend the integrity of exchanged data, protection from eavesdropping, also secured authentication among communication parties. The hardware module implemented in hardware performing as detachment layers among software and hardware elements. Hence these techniques control many parameters of SDR.

Security in cooperative sensing is not only guaranteed by identifying the falsified information due to attacks may come in variety of ways. Which aims in attacking various components and functions of network? The following idea lists the open challenges in cooperative sensing security.

i. Physical Layer Security: Security in physical layer is applied with cooperative sensing which improves security without implementation difficulties of cryptographic capacity. However, it is a challenging task to check the security gain and overhead of physical layer.

ii. Security of Fusion Center: Absolutely this method of security is considered in centralized cooperative sensing methods.

Anyhow, the attacks are susceptible to Fusion Center even though cooperating users have more security. If security is not guaranteed in $\mathrm{FC}$, it is an open issue to cooperative users to recognize the vulnerability and lack of success to Fusion Center (FC). In CR networks, security is regarded as an essential research topic and has drawn attention from academic and industry. CR networks has unique behavior like channel utilization opportunistically and different from wireless networks. A variety of securities have been identified from many years. It could lead to disruption of required operations in CRNs for not solving properly.

The security in a CRN as shown in Fig. 9 may be attacked by the interferences of Malicious Users (MU) in various forms. The attacks from such MU could be defended with strategies like Xiaoyan Wang et al., [94] developed a non-monetary QoS aware auction framework, which resolves secured communication problem for Cognitive Radio Networks. The presented mechanism substantially improves the Primary User privacy rate and generates transmission opportunity for the SU besides avoiding monetary transactions issues. However, effective utilization lowers as the expected transmission rate increases. The network security can thus be modeled into two zones, the privacy zone and the vulnerable zone where the transmitter lies in the former and the receiver falls into the latter at times accompanied by an

eavesdropper as depicted in Fig. 10. In this section, we broadly discussed the security protocols/algorithms for providing security in a Cognitive Radio Network, along with its advantages and disadvantages.

Huifang et al., [95] developed a Joint Spectrum Sensing and Resource Allocation Algorithm (JSSRA), this method supports CRN with SSDF attack. It consists of five modules, namely the JSSRA optimization, cooperative user selection, cooperative spectrum sensing, resource access and SUs trust degree and learning parameter update. The proposed scheme deals with the SSDF attack in CSS to improve the system robustness and achieves a significant gain of the system utility. This algorithm ensures that the SUs reputation degree grows slowly. It makes malicious SUs not to launch the SSDF attack Physical Systems (CPS) users employs spectrum 
sharing in wireless networks. This enhances accuracy of spectrum sensing and protects internal attacks effectively without depending on a central control. However, privacy preservation in this technique is still a concern. Hui et al., [96] developed a Transferring Reputation mechanism and a Dynamic Game based model (TRDG). This technique resolves the reputation loss problem motivated by user's mobility with the help of collaborative spectrum sensing technology. It provides a secure real time response to CyberPhysical Systems (CPS) users employs spectrum sharing in wireless networks. This enhances accuracy of spectrum sensing and protects internal attacks effectively without depending on a central control. However, privacy preservation in this technique is still a concern. Lei et al., [97] studied the downlink security aware resource assignment issue for NonOrthogonal Multiple Access (NOMA) based CRN. It uses $\mathrm{M} / \mathrm{D} / 1$ queuing model to analyze essential secrecy transmission rate for each secondary user. This approach ensures energy efficiency. It is limited to NOMA based CRN.

Mee et al., [98] studied the use of Reinforcement Learning (RL), which promotes to attain optimum results for the enhancement of security by detecting the many malicious nodes and their attacks. The performance enhancements achieved by the intelligent approach of RL including low probability of false positive and missed detection, high detection rate, and utilization gain. Nonetheless, there are several open ends collobarated with the application of RL to security improvement. Xiaoyan et al., [99] introduced truthFul

nONmonetary Double Auction framework (FONDA), which aims to achieve security for PUs communications. This method simplifies starvation of transmission chances for SUs in static

CRNs. This scheme extended to develop d-FONDA to include dynamic CRNs.

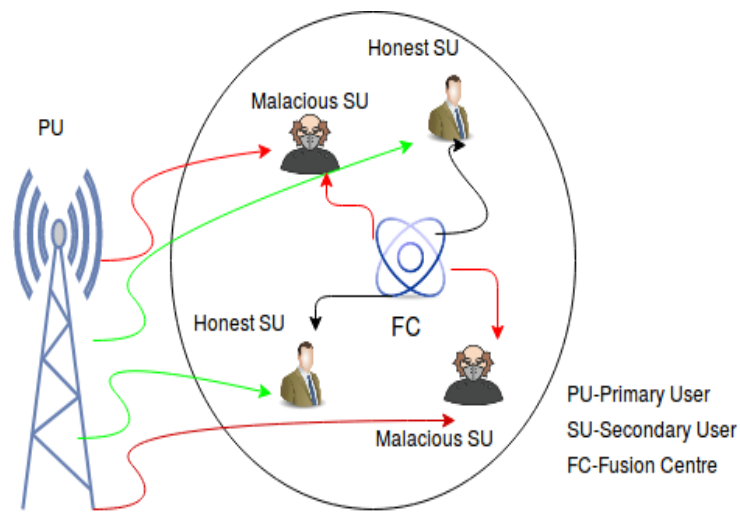

Fig. 9. Privacy in CR networks

Jiliang et al., [100] analyzed the privacy performance of a wireless full-duplex powered Secondary system that transmits in underlay Cognitive Radio Networks. It derives upper and lower bounds probability of Strictly Positive Secrecy Capacity (SPSC). However, these bounds merges to become the probability of SPSC provided exactly the value of interference at the PU receiver is less than that of its interference temperature limit. Lisheng et al., [101] developed a Secure Switch-and-Stay Combining (SSSC) protocol, in which only one of two phase relay is enabled. It supports the transmission security, and relay switches when it can no longer assist the secure communication. This scheme can substantially minimizes the system complexity besides enhancing the opportunistic relaying in the existence of instantaneous or statistical eavesdropping Channel State Information (CSI).
Only it offers a steady network configuration with a lower channel estimation complexity of opportunistic relaying. Hyoungsuk et al., [102] studied a security concern in cooperative scenarios in CRNS, where untrusted SU's facilitate PU's communication in return for the access to the licensed channel. An approach of the information-theoretic secrecy addresses the issue of the unauthorized decoding by the Untrusted SUs. The formulated optimization problem from characterized rate pair achieves secrecy rate of PUs and SUs. The Secondary transmitter dispenses it's transmit powers to achieve maximum data rate during which enables a more secrecy rate to PUs.
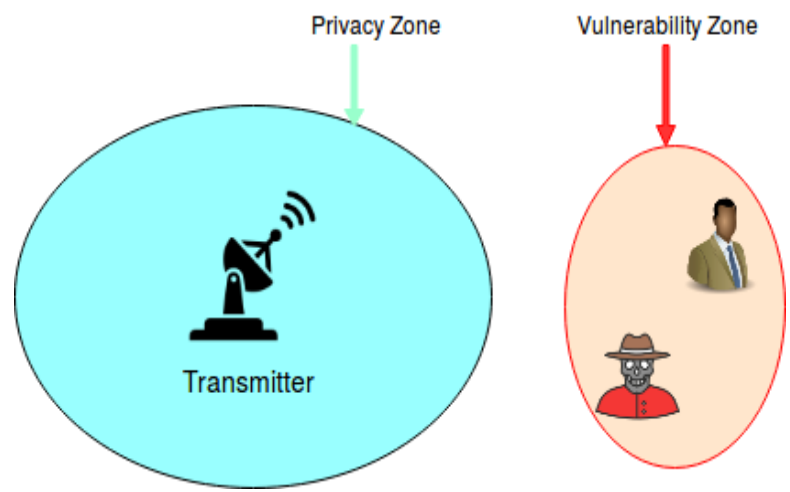

Fig. 10. Network Security

Huifang Chen et al., [103] presented a probabilistic Spectrum Sensing Data Falsification (SSDF) invasion model to illustrate the attacks by malicious SUs. They proposed a robust attackproof Cooperative Spectrum Sensing (CSS)

Huifang Chen et al., [103] presented a probabilistic Spectrum Sensing Data Falsification (SSDF) invasion model to illustrate the attacks by malicious SUs. They proposed a robust attackproof Cooperative Spectrum Sensing (CSS) strategy using M-ary quantized data with a malicious user. Strategy using M-ary quantized data with a malicious user. This eliminates identification method such as intruders from fusion process at Fusion Centre (FC). Even though, there exists a trade-off between performance approach with increased number of intruders and the communication overhead due to higher quantization bits. Yi Li et al., [104] introduced an infrastructure-dependent strategy that relies on available WiFi network to facilitate security and location privacy adopting techniques such as grid reference system and private proximity testing technology. This strategy used as a counter measure for a new way of attack termed as location cheating attack in CRNs driven by databases. However, users' location privacy by trading off service quality causes interferences to PU. Fangwei et al., [105] introduced a dynamic weighted scheme founded on reputation to thwart the various attacks by Malicious Users (MU). This scheme is robust, impedes most of the malicious attacks and has an enhanced detection performance compared to the current schemes. However, this scheme gets more robustness with the number of MUs is less than half the number of all nodes in the network. The following table 3 summarizes the security concepts/algorithm, advantages and disadvantages.

\section{CONCLUSIONS}

In this paper, various techniques in Cognitive Radio Networks have been explored and reviewed for a complete channel utilization which can significantly quench the ever increasing spectrum scarcity issues. We have primarily focused on spectrum management techniques which includes cooperative sensing, spectrum management, spectrum sensing, spectrum 
sharing, spectrum allocation and handoff schemes. A state of art reviews on Energy efficient approaches for routing in CRNs dealing with multi-hop routing and cooperative networks in addition power allocation, energy optimization, resource allocation, energy efficient with harvesting, routing protocols have been addressed Furthermore, we also investigated the security problems arising in such networks and presented effective methods for eliminating the same. A lot of research activities have to be carried out in Spectrum management, Energy Efficient and Security techniques for the enhancement of Cognitive Radio Network communications

Table 3

Security Techniques

\begin{tabular}{|c|c|c|c|c|}
\hline S1.no. & Authors & Concepts/Algorithm & Advantages & Disadvantages \\
\hline 1. & $\begin{array}{l}\text { Huifang et al, (2016) } \\
\text { [95] }\end{array}$ & $\begin{array}{l}\text { Developed a Joint Spectrum Sensing } \\
\text { and Resource Allocation Algorithm } \\
\text { (JSSRA), where this method supports } \\
\text { CRN with SSDF attack. }\end{array}$ & $\begin{array}{l}\text { Improves system robustness } \\
\text { and achieves a significant } \\
\text { gain of the system utility. }\end{array}$ & $\begin{array}{l}\text { It makes malicious SUS dare } \\
\text { not to launch the SSDF } \\
\text { attack. }\end{array}$ \\
\hline 2. & $\begin{array}{l}\text { Lisheng et al., (2016) } \\
\text { [101] }\end{array}$ & $\begin{array}{l}\text { Developed a Secure Switch-and-Stay } \\
\text { Combining (SSSC) protocol, in which } \\
\text { only one of two phase relay is enabled } \\
\text { to support the transmission security, and } \\
\text { relay switches when it can no longer } \\
\text { assist the secure communication. }\end{array}$ & $\begin{array}{l}\text { Substantially minimizes } \\
\text { the system complexity } \\
\text { besides enhances the } \\
\text { opportunistic relaying in } \\
\text { the existence of either the } \\
\text { instantaneous or statistical } \\
\text { eavesdropping Channel } \\
\text { State Information(CSI). }\end{array}$ & $\begin{array}{l}\text { All the links in the } \\
\text { system experience only flat } \\
\text { Rayleigh fading }\end{array}$ \\
\hline 3. & Hui et al., (2017) [96] & $\begin{array}{l}\text { Developed a Transfering Reputation } \\
\text { mechanism and a Dynamic Game based } \\
\text { model (TRDG). }\end{array}$ & $\begin{array}{lrr}\begin{array}{l}\text { Enhances } \\
\text { spectrum accuracy }\end{array} & \begin{array}{r}\text { of } \\
\text { sensing } \\
\text { and }\end{array} \\
\begin{array}{l}\text { protects internal attacks } \\
\text { effectively } \\
\text { depending on a }\end{array} \\
\begin{array}{l}\text { control. } \\
\text { conthout }\end{array} \\
\end{array}$ & $\begin{array}{l}\text { Privacy preservation in this } \\
\text { technique is still a concern. }\end{array}$ \\
\hline 4. & $\begin{array}{l}\text { Xiaoyan et al., } \\
\text { [99] }\end{array}$ & $\begin{array}{l}\text { Introduced truthFulnONmonetary } \\
\text { Double Auction framework (FONDA), } \\
\text { which aims to achieve security for PUs } \\
\text { communications. }\end{array}$ & $\begin{array}{l}\text { Simplifies starvation } \\
\text { transmission chances } \\
\text { SUs in a static CRNs. }\end{array}$ & $\begin{array}{l}\text { The cost of performance } \\
\text { degrades over ideal } \\
\text { schemes. }\end{array}$ \\
\hline 5. & $\begin{array}{l}\text { Huifang et al., (2017) } \\
\text { [103] }\end{array}$ & $\begin{array}{l}\text { Probabilistic Spectrum Sensing Data } \\
\text { Falsification (SSDF) invasion model to } \\
\text { illustrate the attacks by malicious SUs. }\end{array}$ & $\begin{array}{l}\text { Eliminate intruders from } \\
\text { fusion process at Fusion } \\
\text { Centre (FC) }\end{array}$ & $\begin{array}{l}\text { There exists a trade-off } \\
\text { between performance } \\
\text { approach with increased } \\
\text { number of intruders and the } \\
\text { communication overhead } \\
\text { due to higher quantization } \\
\text { bits. }\end{array}$ \\
\hline
\end{tabular}

\section{REFERENCES}

[1] H. Ding, Y. Fang, X. Huang, M. Pan, P. Li, and S. Glisic, "Cognitive Capacity Harvesting Networks: Architectural Evolution Toward Future Cognitive Radio Networks," IEEE Communications Surveys \& Tutorials, vol. 19, no. 3, pp. 1902-1923, 2017.

[2] E. Ahmed, A. Gani, S. Abolfazli, L. J. Yao, and S. U. Khan, "Channel Assignment Algorithms in Cognitive Radio Networks: Taxonomy, Open Issues, and Challenges," IEEE Com Surveys \& Tutorials, vol. 19, no. 3, pp. 1902-1923, 2017.

[3] Y.-C. Liang, K.-C. Chen, G. Y. Li, and P. Mähönen, "Cognitive Radio Networking and Communications: An Overview," IEEE transactions on vehicular technology, vol. 60, no. 7, pp. 3386-3407, 2011.

[4] G. Gür and F. Alagöz, "Green Wireless Communications via Cognitive Dimension: An Overview," IEEE network, no. 2,2011 .

[5] A. A. Khan, M. H. Rehmani, and M. Reisslein, "Cognitive Radio For Smart Grids: Survey of Architectures, Spectrum Sensing Mechanisms, and Networking Protocols," IEEE Communications Surveys \& Tutorials, vol. 18 , no. 1, pp. 860-898, 2016

[6] M. A. Matin, Spectrum Access and Management for Cognitive Radio Networks. Springer, 2017

[7] A. Ali and W. Hamouda, "Advances on Spectrum Sensing for Cognitive Radio Networks: Theory and Applications," IEEE Communications Surveys \& Tutorials, vol. 19, no. 2, pp. 1277-1304, 2017.
[8] S. Atapattu, C. Tellambura, and H. Jiang, Energy Dection for Spectrum Sensing in Cognitive Radio. Springer, 2014.

[9] A. A. Sharifi, M. Sharifi, and M. J. M. Niya, "Secure Cooperative Spectrum Sensing under Primary User Emulation Attack in Cognitive Radio Networks: AttackAware Threshold Selection Approach," AEUInternational Journal of Electronics and Communications, vol. 70, no. 1, pp. 95-104, 2016.

[10] M. Fahimi and A. Ghasemi, "A Distributed Learning Automata Scheme for Spectrum Management in SelfOrganized Cognitive Radio Network," IEEE Transactions on Mobile Computing, vol. 16, no. 6, pp. 1490-1501, 2017.

[11] R. K. Sharma and D. B. Rawat, "Advances on Security Threats and Countermeasures for Cognitive Radio Networks: A survey," IEEE Communications Surveys \& Tutorials, vol. 17, no. 2, pp. 1023-1043, 2015.

[12] H. Reyes, S. Subramaniam, N. Kaabouch, and W. C. Hu, "A Spectrum Sensing Technique Based on Autocorrelation and Euclidean Distance and Its Comparison with Energy Detection for Cognitive Radio Networks," Computers \& Electrical Engineering, vol. 52, pp. 319-327, 2016.

[13] J. Lai, E. Dutkiewicz, R. P. Liu, and R. Vesilo, "Opportunistic Spectrum Access with Two Channel Sensing in Cognitive Radio Networks," IEEE Transactions on Mobile Computing, vol. 14, no. 1, pp. $126-138,2015$.

[14] A. Singh, M. R. Bhatnagar, and R. K. 
Mallik,"Performance of an Improved Energy Detector in Multihop Cognitive Radio Networks," IEEE transactions on Vehicular technology, vol. 65, no. 2, pp. 732-743, 2016.

[15] T. Xiong, Z. Li, Y.-D. Yao, and P. Qi, "Random, Persistent, and Adaptive Spectrum Sensing Strategies for Multiband Spectrum Sensing in Cognitive Radio Networks with Secondary User Hardware Limitation," IEEE Access, vol. 5, pp. 14 854-14 866, 2017.

[16] D. Wang, N. Zhang, Z. Li, F. Gao, and X. Shen, "Leveraging High Order Cumulants for Spectrum Sensing and Power Recognition in Cognitive Radio Networks," IEEE Transactions on Wireless Communications, vol. 17, no. 2, pp. 1298-1310, 2018.

[17] J. Zhao, Q. Liu, X. Wang, and S. Mao, "Scheduling of Collaborative Sequential Compressed Sensing over Wide Spectrum Band," IEEE/ACM Transactions on Networking (TON), vol. 26, no. 1, pp. 492-505, 2018.

[18] W. Cheng, X. Zhang, and H. Zhang, "Full-Duplex Spectrum-Sensing and Mac-Protocol for Multichannel Nontime-Slotted Cognitive Radio Networks," IEEE Journal on Selected Areas in Communications, vol. 33, no. 5, pp. 820-831, 2015.

[19] Z. Wei, Z. Feng, Q. Zhang, and W. Li, "Three Regions for Space-Time Spectrum Sensing and Access in Cognitive Radio Networks," IEEE Transactions on Vehicular Technology, vol. 64, no. 6, pp. 2448-2462, 2015

[20] W. Na, J. Yoon, S. Cho, D. W. Griffith, and N. Golmie, "Centralized Cooperative Directional Spectrum Sensing for Cognitive Radio Networks," IEEE Transactions on Mobile Computing, 2017

[21] A. Patel, H. Ram, A. K. Jagannatham, and P. K. Varshney, "Robust Cooperative Spectrum Sensing for MIMO Cognitive Radio Networks Under CSI Uncertainty," IEEE Transactions on Signal Processing, vol. 66, no. 1, pp. 18-33, 2018.

[22] T. Xue, X. Dong, and Y. Shi, "Resource-Allocation Strategy for Multiuser Cognitive Radio Systems: Location-Aware Spectrum Access," IEEE Transactions on Vehicular Technology, vol. 66, no. 1, pp. 884-889, 2017.

[23] X. Yuan, C. Jiang, Y. Shi, Y. T. Hou, W. Lou, S. Kompella, and S. F. Midkiff, "Toward Transparent Coexistence for Multihop Secondary Cognitive Radio Networks," IEEE Journal on Selected Areas in Communications, vol. 33, no. 5, pp. 958-971, 2015.

[24] S. Chen, Z. Zeng, and C. Guo, "Polarization and Power Optimization for Spectrum Sharing in Cognitive Heterogeneous Cellular Network," pp. 1-6, 2017.

[25] A. Saha, S. S. Bhattacherjee, C. K. De, and D. De, "Cooperative Spectrum Sharing with Multi-Antenna Based Adaptive Hybrid Relay in Presence of Multiple Primary Users," Journal of Information and Optimization Sciences, vol. 38, no. 6, pp. 857-871, 2017.

[26] R.-z. Wu, L.-y. Gao, L.-r. Tang, and J.-j. Zhu, "Optimal Spectrum Allocation of Cognitive Radio Network Under Underlay Model," Wireless Personal Communications, vol. 97, no. 1, pp. 469-481, 2017.
[27] A. Lertsinsrubtavee and N. Malouch, "Hybrid Spectrum Sharing Through Adaptive Spectrum Handoff and Selection," IEEE transactions on mobile computing, vol. 15, no. 11, pp. 2781-2793, 2016.

[28] V.-D. Nguyen and O.-S. Shin, "Cooperative Predictionand-Sensing-Based Spectrum Sharing in Cognitive Radio Networks," IEEE Transactions on Cognitive Communications and Networking, vol. 4, no. 1, pp. 108$120,2018$.

[29] J. Lundén, M. Motani, and H. V. Poor, "Distributed Algorithms for Sharing Spectrum Sensing Information in Cognitive Radio Networks," IEEE Transactions on Wireless Communications, vol. 14, no. 8, pp. 4667-4678, 2015.

[30] X. Feng, G. Sun, X. Gan, F. Yang, X. Tian, X. Wang, and M. Guizani, "Cooperative Spectrum Sharing in Cognitive Radio Networks: A Distributed Matching Approach," IEEE Transactions on Communications, vol. 62, no. 8, pp. 2651-2664, 2014.

[31] Z. Shu, Y. Qian, and R. Q. Hu, "Delay Based Channel Allocations in Multi-Hop Cognitive Radio Networks," pp. 1475-1480, 2015.

[32] S. Aslam, A. Shahid, and K.-G. Lee, "Primary User Behavior Aware Spectrum Allocation Scheme for Cognitive Radio Networks," Computers \& Electrical Engineering, vol. 42, pp. 135-147, 2015.

[33] M. Hawa, A. AlAmmouri, A. Alhiary, and N. Alhamad, "Distributed Opportunistic Spectrum Sharing in Cognitive Radio Networks," international journal of communication systems, vol. 30, no. 7, pp 31--47, 2017.

[34] M. Yousefvand, N. Ansari, and S. Khorsandi, "Maximizing Network Capacity of Cognitive Radio Networks by Capacity-Aware Spectrum Allocation," IEEE Transactions on Wireless Communications, vol. 14, no. 9, pp. 5058-5067, 2015.

[35] P. Maheshwari and A. K. Singh, "A Fuzzy Logic Based Approach to Spectrum Assignment in Cognitive Radio Networks," pp. 278-281, 2015.

[36] S. Manjula, C. Abhilash, K. Shaila, K. Venugopal, and L. Patnaik, "Performance of AODV Routing Protocol using Group and Entity Mobility Models in Wireless Sensor Networks," Proceedings of the International MultiConference of Engineers and Computer Scientists, vol. 2, pp. 1212-1217, 2008.

[37] A. F. Tayel, S. I. Rabia, and Y. Abouelseoud, "An Optimized Hybrid Approach for Spectrum Handoff in Cognitive Radio Networks with Non-Identical Channels," IEEE transactions on communications, vol. 64, no. 11, pp. 4487-4496, 2016.

[38] R. Alhamad, H. Wang, and Y.-D. Yao, "Cooperative Spectrum Sensing with Random Access Reporting Channels in Cognitive Radio Networks," IEEE Transactions on Vehicular Technology, vol. 66, no. 8, pp. 7249-7261, 2017.

[39] Y. Wu, Q. Yang, X. Liu, and K. S. Kwak, "DelayConstrained Optimal Transmission with Proactive Spectrum Handoff in Cognitive Radio Networks," IEEE Transactions on Communications, vol. 64, no. 7, pp. 2767-2779, 2016. 
[40] Y. Wu, F. Hu, S. Kumar, Y. Zhu, A. Talari, N. Rahnavard, and J. D. Matyjas, "A Learning-Based QoE-Driven Spectrum Handoff Scheme for Multimedia Transmissions over Cognitive Radio Networks," IEEE Journal on Selected Areas in Communications, vol. 32, no. 11, pp. 2134-2148, 2014.

[41] A. O. Bicen, E. B. Pehlivanoglu, S. Galmes, and O. B. Akan, "Dedicated Radio Utilization for Spectrum Handoff and Efficiency in Cognitive Radio Networks," IEEE Transactions on Wireless Communications, vol. 14, no. 9, pp. 5251-5259, 2015.

[42] W. Zhang and C. K. Yeo, "Sequential Sensing Based Spectrum Handoff in Cognitive Radio Networks with Multiple Users," Computer Networks, vol. 58, pp. 8798, 2014.

[43] U. Prathap, P. D. Shenoy, K. Venugopal, and L. Patnaik, "Wireless Sensor Networks Applications and Routing Protocols: Survey and Research Challenges," Cloud and Services Computing (ISCOS), 2012 International Symposium on, pp. 49-56, 2012.

[44] C. Li, Z. Liu, X. Geng, M. Dong, F. Yang, X. Gan, X. Tian, and X. Wang, "Two Dimension Spectrum Allocation for Cognitive Radio Networks," IEEE Transactions on Wireless Communications, vol. 13, no. 3, pp. 1410-1423, 2014

[45] J. Zou, Q. Wu, H. Xiong, and C. W. Chen, "Dynamic Spectrum Access and Power Allocation for Cooperative Cognitive Radio Networks," IEEE Transactions on Signal Processing, vol. 63, no. 21, pp. 5637-5649, 2015.

[46] Y. Zhang, W. P. Tay, K. H. Li, and D. Gaïti, "Distributed Boundary Estimation for Spectrum Sensing in Cognitive Radio Networks," IEEE Journal on Selected Areas in Communications, vol. 32, no. 11, pp. 1961-1973, 2014.

[47] S. Tarannum, S. Srividya, D. Asha, R. Padmini, L. Nalini,K. Venugopal, and L. Patnaik, "Dynamic Hierarchical Communication Paradigm for Wireless Sensor Networks: A Centralized, Energy Efficient Approach," Communication Systems, 2008. ICCS 2008. $11^{\text {th }}$ IEEE Singapore International Conference on, pp. 959-963, 2008.

[48] M. R. Amini, M. Mahdavi, and M. J. Omidi, "Energy Efficiency Optimization of Secondary Network Considering Primary User Return with AlternatingPhase-Type Traffic," IEEE Transactions on Communications, vol. 65, no. 7, pp. 3095-3109, 2017.

[49] A. Furtado, L. Irio, R. Oliveira, L. Bernardo, and R. Dinis, "Spectrum Sensing Performance in Cognitive Radio Networks with Multiple Primary Users," IEEE Transactions on Vehicular Technology, vol. 65, no. 3, pp. 1564-1574, 2016.

[50] M. Yousefvand, T. Han, N. Ansari, and A. Khreishah, "Distributed Energy-Spectrum Trading in Green Cognitive Radio Cellular Networks," IEEE Transactions on Green Communications and Networking, vol. 1, no. 3 , pp. 253-263, 2017.

[51] K. Kiran, A. A. Singh, P. D. Shenoy, K. Venugopal, and L. M. Patnaik, "Analysis of Traffic Splitting over A Multi-Hop Network with Hybrid Wimax and Wifi Nodes," Parallel Distributed and Grid Computing (PDGC), 2012 2nd IEEE International Conference on, pp. 609-613, 2012.
[52] M. Usman, D. Har, and I. Koo, "Energy-Efficient Infrastructure Sensor Network for Ad Hoc Cognitive Radio Network," IEEE Sensors Journal, vol. 16, no. 8, pp. 2775-2787, 2016.

[53] M. Brown, C. Marshall, D. Yang, M. Li, J. Lin, and G. Xue, "Maximizing Capacity in Cognitive Radio Networks under Physical Interference Model," IEEE/ACM Transactions on Networking, vol. 25, no. 5, pp. 3003-3015, 2017.

[54] C. X. Mavromoustakis, G. Mastorakis, A. Bourdena, and E. Pallis, "Energy-Efficient Management using A TrafficOriented Routing Scheme for Cognitive Radio Networks," International Journal of Network Management, vol. 25, no. 6, pp. 418-434, 2015.

[55] P. Rathika and S. Sophia, "A Distributed Scheduling Approach for Qos Improvement in Cognitive Radio Networks," Computers \& Electrical Engineering, vol. 57, pp. 186-198, 2017.

[56] M. Tabassum, M. A. Razzaque, M. N. S. Miazi, M. M. Hassan, A. Alelaiwi, and A. Alamri, "An Energy Aware Event-Driven Routing Protocol for Cognitive Radio Sensor Networks," Wireless Networks, vol. 22, no. 5, pp. 1523-1536, 2016.

[57] R. Mochaourab, B. Holfeld, and T. Wirth, "Distributed Channel Assignment in Cognitive Radio Networks: Stable Matching and Walrasian Equilibrium," IEEE Transactions on Wireless Communications, vol. 14, no. 7, pp. 3924-3936, 2015.

[58] J. Liu, H. Ding, Y. Cai, H. Yue, Y. Fang, and S. Chen, "An Energy-Efficient Strategy for Secondary Users in Cooperative Cognitive Radio Networks for Green Communications," IEEE Journal on Selected Areas in Communications, vol. 34, no. 12, pp. 3195-3207, 2016.

[59] T. Suzuki and T. Fujii, "Joint Routing and Spectrum Allocation for Multi-hop Inter-Vehicle Communication in Cognitive Radio Networks,” International Journal of Intelligent Transportation Systems Research, vol. 15, no. 1, pp. 39-49, 2017.

[60] L. Zhang, G. Zhao, W. Zhou, L. Li, G. Wu, Y.-C. Liang, and S. Li, "Primary Channel Gain Estimation for Spectrum Sharing in Cognitive Radio Networks," IEEE Transactions on Communications, vol. 65, no. 10, pp. 4152-4162, 2017.

[61] M. Laghate and D. Cabric, "Cooperatively Learning Footprints of Multiple Incumbent Transmitters by Using Cognitive Radio Networks," IEEE Transactions on Cognitive Communications and Networking, vol. 3, no. 3, pp. 282-297, 2017.

[62] H. Cao, H. Tian, J. Cai, A. S. Alfa, and S. Huang, "Dynamic Load-Balancing Spectrum Decision for Heterogeneous Services Provisioning in Multi-Channel Cognitive Radio Networks," IEEE Transactions on Wireless Communications, vol. 16, no. 9, pp. 5911-5924, 2017.

[63] H. Zhang, C. Jiang, N. C. Beaulieu, X. Chu, X. Wang, and T. Q. Quek, "Resource Allocation for Cognitive Small Cell Networks: A Cooperative Bargaining Game Theoretic Approach," IEEE Transactions on Wireless Communications, vol. 14, no. 6, pp. 3481-3493, 2015.

[64] P. Bhardwaj, A. Panwar, O. Ozdemir, E. Masazade, I. 
Kasperovich, A. L. Drozd, C. K. Mohan, and P. K. Varshney, "Enhanced Dynamic Spectrum Access in Multiband Cognitive Radio Networks via Optimized Resource Allocation," IEEE Transactions on Wireless Communications, vol. 15, no. 12, pp. 8093-8106, 2016.

[65] D. Zhang, Z. Chen, J. Ren, N. Zhang, M. K. Awad, H. Zhou, and X. S. Shen, "Energy-Harvesting-Aided Spectrum Sensing and Data Transmission in Heterogeneous Cognitive Radio Sensor Network," IEEE Transactions on Vehicular Technology, vol. 66, no. 1, pp. 831-843, 2017.

[66] J. Van Hecke, P. Del Fiorentino, V. Lottici, F. Giannetti, L. Vandendorpe, and M. Moeneclaey, "Distributed Dynamic Resource Allocation for Cooperative Cognitive Radio Networks with Multi-Antenna Relay Selection," IEEE Transactions on Wireless Communications, vol. 16, no. 2, pp. 1236-1249, 2017.

[67] N. Zhao, F. R. Yu, H. Sun, and M. Li, "Adaptive Power Allocation Schemes for Spectrum Sharing in Interference-Alignment-Based Cognitive Radio Networks," IEEE transactions on vehicular technology, vol. 65 , no. 5, pp. 3700-3714, 2016.

[68] H. Hu, H. Zhang, and N. Li, "Location-InformationAssisted Joint Spectrum Sensing and Power Allocation for Cognitive Radio Networks with Primary-User Outage Constraint," IEEE Transactions on Vehicular Technology, vol. 65 , no. 2, pp. 658-672, 2016.

[69] K. Singh and S. Moh, "An Energy-Efficient and Robust Multipath Routing Protocol for Cognitive Radio Ad Hoc Networks," Sensors, vol. 17, no. 9, p. 2027, 2017.

[70] Z. Yang, Y. Gu, Z. Wu, N. Zhao, and X. Wang, "An Energy-Efficient Routing Protocol for Cognitive Radio Enabled AMI Networks in Smart Grid,” pp. 1-5, 2017.

[71] J. Wang, H. Yue, L. Hai, and Y. Fang, "Spectrum-Aware Anypath Routing in Multi-Hop Cognitive Radio Networks," IEEE Transactions on Mobile Computing, vol. 16, no. 4, pp. 1176-1187, 2017.

[72] W. Xu, W. Yuan, Q. Shi, X. Wang, and Y. Zhang, "Distributed Energy-Efficient Cross-Layer Optimization for Multihop MIMO Cognitive Radio Networks With Primary User Rate Protection," IEEE Transactions on Vehicular Technology, vol. 66, no. 1, pp. 785-797, 2017

[73] G. A. Shah, F. Alagoz, E. A. Fadel, and O. B. Akan, "A Spectrum-Aware Clustering for Efficient Multimedia Routing in Cognitive Radio Sensor Networks," IEEE Transactions on Vehicular Technology, vol. 63, no. 7, pp. 3369-3380, 2014.

[74] T. S. Prakash, G. Badrinath, K. Venugopal, and L. M. Patnaik, "Energy Aware Topology Management in Ad Hoc Wireless Networks," Systems and Networks Communications, 2006. ICSNC'06. International Conference on, pp. 1-1, 2006.

[75] M. E. Ahmed, D. I. Kim, and K. W. Choi, "Traffic-aware optimal spectral Access in wireless powered cognitive radio networks," IEEE Transactions on Mobile Computing, vol. 17, no. 3, pp. 733-745, 2018.

[76] S. Tarannum, B. Aravinda, L. Nalini, K. Venugopal, and L. Patnaik, "Routing Protocol for Lifetime Maximization of Wireless Sensor Networks," Advanced Computing and Communicatons, 2006. ADCOM 2006. International
Conference on, pp. 401-406, 2006.

[77] Z. Li, B. Liu, J. Si, and F. Zhou, "Optimal Spectrum Sensing Interval in Energy-Harvesting Cognitive Radio Networks," IEEE Transactions on Cognitive Communications and Networking, vol. 3, no. 2, pp. 190200, 2017

[78] A. Kanavalli, D. Sserubiri, P. D. Shenoy, K. Venugopal, and L. Patnaik, "A Flat Routing Protocol for Sensor Networks," Methods and Models in Computer Science, 2009. ICM2CS 2009. Proceeding of International Conference on, pp. 1-5, 2009

[79] H. Xing, X. Kang, K.-K. Wong, and A. Nallanathan, "Optimizing DF Cognitive Radio Networks with FullDuplex-Enabled Energy Access Points," IEEE Transactions on Wireless Communications, vol. 16, no. 7, pp. 4683-4697, 2017.

[80] S. Vahidian, E. Soleimani-Nasab, S. Aïssa, and M. Ahmadian-Attari, "Bidirectional AF Relaying with Underlay Spectrum Sharing in Cognitive Radio Networks," IEEE Transactions on Vehicular Technology, vol. 66 , no. 3, pp. 2367-2381, 2017

[81] A. Guirguis, M. Karmoose, K. Habak, M. El-Nainay, and M. Youssef, "Cooperation-based Multi-Hop Routing Protocol for Cognitive Radio Networks," Journal of Network and Computer Applications, vol. 110, pp. 27 42, 2018.

[82] R. Bordon, S. M. Sánchez, S. B. Mafra, R. D. Souza, J. L. Rebelatto, and E. M. G. Fernandez, "Energy Efficient Power Allocation Schemes for a Two-User NetworkCoded Cooperative Cognitive Radio Network." IEEE Trans. Signal Processing, vol. 64, no. 7, pp. 1654-1667, 2016.

[83] S. Ping, A. Aijaz, O. Holland, and A.-H. Aghvami, "SACRP: A Spectrum Aggregation-Based Cooperative Routing Protocol for Cognitive Radio Ad-Hoc Networks," IEEE transactions on communications, vol 63, no. 6, 2015.

[84] Y. Ma, Y. Gao, Y.-C. Liang, and S. Cui, "Reliable and Efficient Sub-Nyquist Wideband Spectrum Sensing in Cooperative Cognitive Radio Networks," IEEE Journal on Selected Areas in Communications, vol. 34, no. 10, pp. 2750-2762, 2016.

[85] D. Jiang, X. Ying, Y. Han, and Z. Lv, "Collaborative Multi-Hop Routing in Cognitive Wireless Networks,' Wireless personal communications, vol. 86, no. 2, pp. 901-923, 2016.

[86] Y. Liu and L. Dong, "Spectrum Sharing in MIMO Cognitive Radio Networks based on Cooperative Game Theory," IEEE Transactions on Wireless Communications, vol. 13, no. 9, pp. 4807-4820, 2014

[87] D. Jiang, Z. Xu, and Z. Lv, "A Multicast Delivery Approach with Minimum Energy Consumption for Wireless Multi-Hop Networks," Telecommunication systems, vol. 62, no. 4, pp. 771-782, 2016.

[88] Y. Song, J. Xie, and X. Wang, "A Novel Unified Analytical Model for Broadcast Protocols in Multi-Hop Cognitive Radio Ad Hoc Networks," IEEE Transactions on Mobile Computing, vol. 13, no. 8, pp. 1653-1667, 2014. 
[89] D. Das and A. A. Abouzeid, "Delay Analysis of Multihop Cognitive Radio Networks Using Network of Virtual Priority Queues," pp. 1514-1519, 2014.

[90] A. Banerjee, A. Paul, and S. P. Maity, "Joint Power Allocation and Route Selection for Outage Minimization in Multihop Cognitive Radio Networks with Energy Harvesting," IEEE Transactions on Cognitive Communications and Networking, vol. 4, no. 1, pp. 8292, 2018.

[91] Q. Liang, X. Wang, X. Tian, F. Wu, and Q. Zhang, “TwoDimensional Route Switching in Cognitive Radio Networks: A Game-Theoretical Framework," IEEE/ACM Transactions on Networking, vol. 23, no. 4, pp. 1053-1066, 2015.

[92] S. Parvin, F. K. Hussain, O. K. Hussain, S. Han, B. Tian, and E. Chang, "Cognitive Radio Network Security: A Survey," Journal of Network and Computer Applications, vol. 35 , no. 6, pp. 1691-1708, 2012.

[93] A. G. Fragkiadakis, E. Z. Tragos, I. G. Askoxylakis et al., "A Survey on Security Threats and Detection Techniques in Cognitive Radio Networks," IEEE Communications Surveys \& Tutorials, vol. 15, no. 1, pp. 428-445, 2013

[94] X. Wang, Y. Ji, H. Zhou, and J. Li, "A Nonmonetary Qos-Aware Auction Framework Toward Secure Communications for Cognitive Radio Networks," IEEE Transactions on Vehicular Technology, vol. 65, no. 7, pp. 5611-5623, 2016.

[95] H. Chen, M. Zhou, L. Xie, K. Wang, and J. Li, "Joint Spectrum Sensing and Resource Allocation Scheme in Cognitive Radio Networks with Spectrum Sensing Data Falsification Attack," IEEE Transactions on Vehicular Technology, vol. 65, no. 11, pp. 9181-9191, 2016.

[96] H. Lin, J. Hu, J. Ma, L. Xu, and Z. Yu, "A Secure Collaborative Spectrum Sensing Strategy in CyberPhysical Systems," IEEE Access, vol. 5, pp. 27 679-27 690, 2017.

[97] L. Xu, A. Nallanathan, X. Pan, J. Yang, and W. Liao, "Security-Aware Resource Allocation with Delay Constraint for NOMA-Based Cognitive Radio Network," IEEE Transactions on Information Forensics and
Security, vol. 13, no. 2, pp. 366-376, 2018.

[98] M. H. Ling, K.-L. A. Yau, J. Qadir, G. S. Poh, and Q. Ni, "Application of Reinforcement Learning for Security Enhancement in Cognitive Radio Networks," Applied Soft Computing, vol. 37, pp. 809-829, 2015.

[99] X. Wang, Y. Ji, H. Zhou, and J. Li, "Auction-Based Frameworks for Secure Communications in Static and Dynamic Cognitive Radio Networks," IEEE Transactions on Vehicular Technology, vol. 66, no. 3, pp. 2658-2673, 2017.

[100] J. Zhang, G. Pan, and H.-M. Wang, "On PhysicalLayer Security in Underlay Cognitive Radio Networks with Full-Duplex Wireless-Powered Secondary System," IEEE Access, vol. 4, pp. 3887-3893, 2016.

[101] L. Fan, S. Zhang, T. Q. Duong, and G. K. Karagiannidis, "Secure Switch-and-Stay Combining (SSSC) for Cognitive Relay Networks," IEEE Transactions on Communications, vol. 64 , no. 1 , pp. 70 82, 2016.

[102] H. Jeon, S. W. McLaughlin, I.-M. Kim, and J. Ha, "Secure Communications with Untrusted Secondary Nodes in Cognitive Radio Networks," IEEE Transactions on Wireless Communications, vol. 13, no. 4, pp. 17901805,2014

[103] H. Chen, M. Zhou, L. Xie, and J. Li, "Cooperative Spectrum Sensing with M-Ary Quantized Data in Cognitive Radio Networks under SSDF Attacks," IEEE Transactions on Wireless Communications, vol. 16, no. 8, pp. 5244-5257, 2017.

[104] Y. Li, L. Zhou, H. Zhu, and L. Sun, "PrivacyPreserving Location Proof for Securing Large-Scale Database-Driven Cognitive Radio Networks," IEEE Internet of Things Journal, vol. 3, no. 4, pp. 563-571, 2016.

[105] L. Fangwei, L. Fan, Z. Jiang, and N. Yifang, "Reputation-Based Secure Spectrum Situation Fusion in Distributed Cognitive Radio Networks," The Journal of China Universities of Posts and Telecommunications, vol. 22 , no. 3, pp. $110-117,2015$ 